Provided for non-commercial research and education use. Not for reproduction, distribution or commercial use.

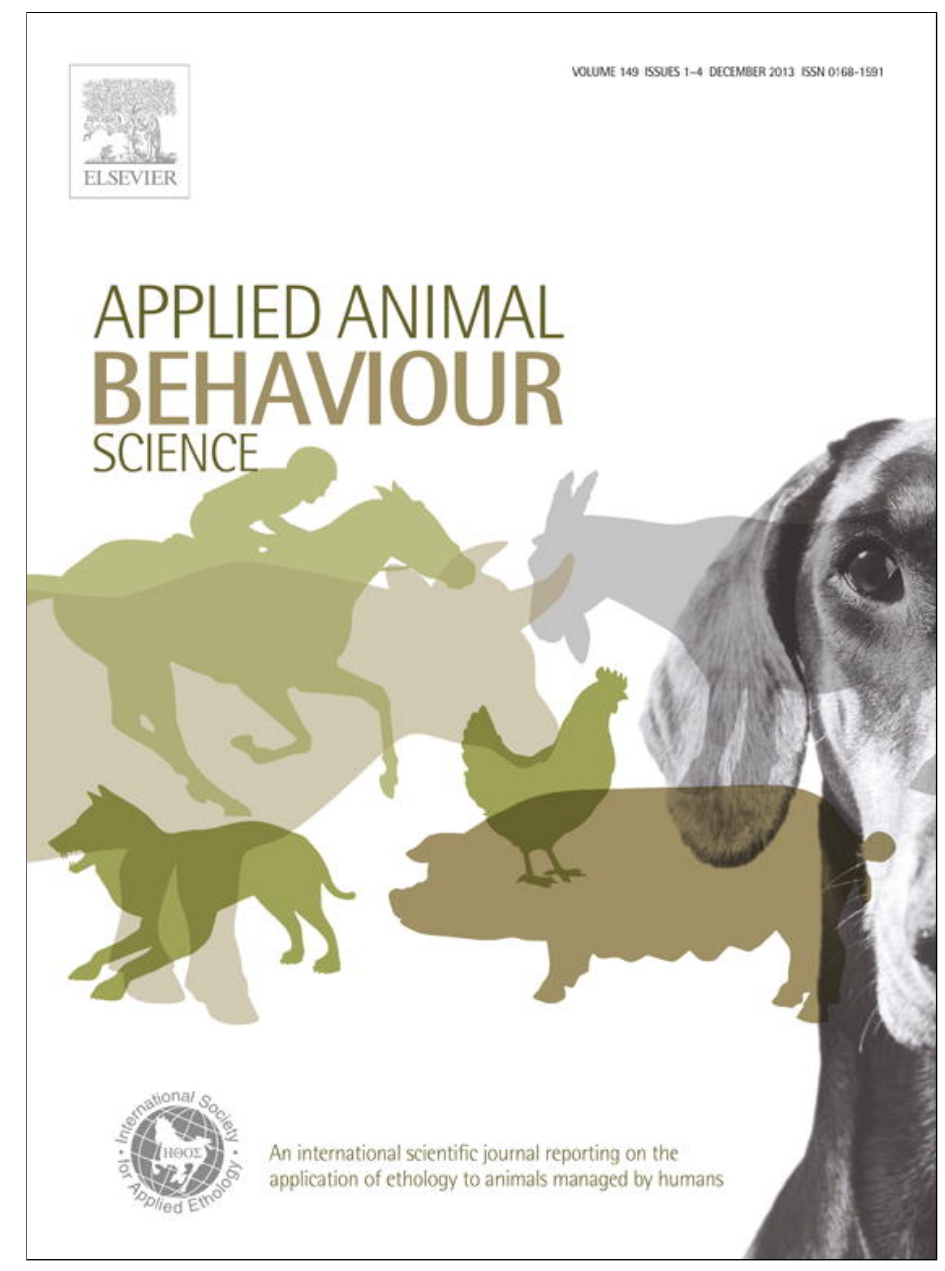

This article appeared in a journal published by Elsevier. The attached copy is furnished to the author for internal non-commercial research and education use, including for instruction at the authors institution and sharing with colleagues.

Other uses, including reproduction and distribution, or selling or licensing copies, or posting to personal, institutional or third party websites are prohibited.

In most cases authors are permitted to post their version of the article (e.g. in Word or Tex form) to their personal website or institutional repository. Authors requiring further information regarding Elsevier's archiving and manuscript policies are encouraged to visit:

http://www.elsevier.com/authorsrights 


\title{
Efficacy of foraging enrichments to increase foraging time in Grey parrots (Psittacus erithacus erithacus)
}

\author{
Yvonne R.A. van Zeeland*, Nico J. Schoemaker, Manon M. Ravesteijn, \\ Marjon Mol, Johannes T. Lumeij
}

Division of Zoological Medicine, Department of Clinical Sciences of Companion Animals, Faculty of Veterinary Medicine, Utrecht University, Utrecht, The Netherlands

\section{A R T I C L E I N F O}

\section{Article history:}

Accepted 17 September 2013

Available online 25 September 2013

\section{Keywords:}

Animal welfare

Environmental enrichment

Foraging

Foraging enrichment

Psittacine

Parrot

\begin{abstract}
A B S T R A C T
Foraging enrichment is considered one of the most effective strategies to improve welfare and reduce stereotypies and other abnormal repetitive behaviours in captive animals, including parrots. Few studies, however, have investigated the effects of the different types of enrichment and determined their effect on increasing foraging times and foraging-related activities.

To study the effect of different types of enrichment on foraging activity, eleven types of foraging enrichment [multiple bowls, mixing food with inedible items, larger-sized food particles, and 8 puzzle feeders] were tested in 12 Grey parrots (Psittacus erithacus erithacus). After acclimatization and assessment of baseline foraging times, enrichments were presented in a random order. Video recordings were used to analyze total foraging times and times spent on the different foraging activities. In addition, the frequencies and duration of foraging bouts, and the times at which they occurred, were determined. The learning curves and presence of habituation to the enrichment over a 1-week period were also assessed.

Parrots needed $8.3 \pm 1.1$ days to learn how to use the foraging enrichments. For two of the puzzle feeders, it took considerably longer. Nine out of eleven foraging enrichments were able to significantly increase foraging times, with the most effective enrichments resulting in a 2- to 2.5 -fold increase compared to baseline values ( $47 \pm 18 \mathrm{~min}$ ). The increases in time spent on foraging could be attributed to an increased amount of time spent on enrichmentrelated activities and/or an increased amount of time spent on food-related activities. None of the enrichments (including the use of multiple bowls in different locations) resulted in significant increases in time spent on locomotory behaviours (i.e. movement towards or away from the feeding site). No significant downwards trends in foraging times were observed over the 1-week observation period. The duration of the observations may, however, have been too short to demonstrate habituation effects.

Results of this study show that distinct differences are present in the difficulty level, efficacy and time allocation, thereby affecting the choice for a particular foraging enrichment. Although most of the foraging enrichments were able to significantly increase foraging time, none of the enrichments were able to increase foraging times to levels comparable with that of wild conspecifics (i.e. 4-6 hours per day). New, more effective types of foraging enrichment should therefore be developed and tested.
\end{abstract}

(c) 2013 Elsevier B.V. All rights reserved.

* Corresponding author at: Division of Zoological Medicine, Department of Clinical Sciences of Companion Animals, Faculty of Veterinary Medicine, Utrecht University, Yalelaan 108, 3584 CM Utrecht, The Netherlands. Tel.: +31 30253 4542; fax: +31 302518126.

E-mail addresses: Y.R.A.vanZeeland@uu.nl, y_van_zeeland@hotmail.com (Y.R.A.van Zeeland). 


\section{Introduction}

In the wild, most animals, including parrots, spend an important part of their daily activity on foraging. Many parrot species regularly travel several miles between feeding sites in search of food (e.g., Snyder et al., 1987; Gilardi and Munn, 1998; Wirminghaus et al., 2001; Symes and Perrin, 2003). Once they arrive at a feeding site, they typically engage in a wide variety of foraging behaviours, including local search for food, food selection and procurement, food manipulation and food consumption (e.g., Magrath and Lill, 1985; Snyder et al., 1987; May, 2001). Depending on the species and season, the time invested on these behaviours may vary from 40 to $75 \%$ of the daytime, i.e., $4-8$ hours/day (Magrath and Lill, 1985; Snyder et al., 1987; Westcott and Cockburn, 1988; Birchall, 1990; Renton, 2001; Francisco et al., 2002).

Captive parrots, on the other hand, do not need to travel large distances, visit multiple food sites and/or search and select their food. In addition, they often have little to no opportunity to manipulate objects to obtain their food (Bauck, 1998). As a consequence, captive parrots usually spend less than an hour on feeding (Oviatt and Millam, 1997; Rozek et al., 2010). Their instincts, behaviour and needs, however, are likely to be the same as those of their wild conspecifics, since most parrots are only one or two generations removed from the wild and thus not considered as domesticated species (Davis, 1998; Graham, 1998). Similar to other animal species such as rats, pigs, monkeys, starlings, pigeons and chickens (Inglis et al., 1997; Lindqvist et al., 2002; de Leeuw, 2004; Ogura, 2011), parrots will contrafreeload (i.e., work for food even when food is freely available), suggesting that there is a behavioural need to forage (Coulton et al., 1997; van Zeeland et al., 2009; Joseph, 2010). This behavioural need, which is controlled by both physiological and psychological parameters, can only be satiated if an animal performs and experiences the consequences of both the appetitive and consummatory phases of feeding (Collier and Rovée-Collier, 1981; Hughes and Duncan, 1988; Jensen and Toates, 1993).

The predictability and ease with which food can be obtained in the conventional captive living environment restrict the parrots' opportunities to forage. As a consequence of the inability to carry out this species-specific behaviour, welfare may be reduced and abnormal repetitive behaviours may arise (Rushen et al., 1993; Mason and Mendl, 1997), including oral stereotypies (e.g., wire chewing, tongue playing, food manipulation or dribbling) and feather damaging behaviour, also referred to as feather picking, feather plucking or pterotillomania (Meehan et al., 2003, 2004; Lumeij and Hommers, 2008).

Environmental enrichment may help reduce behavioural problems and provide the animal with opportunities to perform its normal behaviour and cope with its captive living environment (Newberry, 1995; Young, 2003; Swaisgood and Shepherdson, 2005). Foraging enrichment in particular appears to be among the most effective measures (Crocket, 1998; Miller and Mench, 2005; Dixon et al., 2010) as the provision of foraging opportunities in captivity was found to 1 ) increase activity; 2) provide cognitive stimulation and manipulative activities; 3) alleviate stress, frustration and boredom; and 4) reduce and prevent aggression, and abnormal repetitive behaviours, including stereotypies (Bloomsmith et al., 1988; Shepherdson et al., 1993; Spoolder et al., 1995; Baker, 1997; Aerni et al., 2000; Bashaw et al., 2003; Johnson et al., 2004; Honess and Marin, 2006; VargasAshby and Pankhurst, 2007; Brinch-Riber and Mench, 2008). In parrots, provision of foraging enrichments has been associated with positive behavioural changes and welfare effects (Coulton et al., 1997; van Hoek and King, 1997; Meehan et al., 2003, 2004; Elson and Marples, 2001; Lumeij and Hommers, 2008). The development of feather damaging behaviour and stereotypies in Amazon parrots, for example, could significantly be reduced by increasing the physical complexity of the environment and providing parrots with foraging devices (Meehan et al., 2003, 2004). In addition, foraging enrichment was found to improve feather score and foraging time in Grey parrots (Lumeij and Hommers, 2008).

Over the years, several approaches have been developed to stimulate foraging behaviour and increase foraging times. These include 1 ) provision of smaller, more frequent meals in multiple locations (i.e. increase spatial or temporal variability of feeding); 2) scatter or hide food in the enclosure (i.e. increase search time); 3 ) provision of live prey (i.e. increase capture time); 4) provision of foraging devices or puzzle feeders (i.e. increase extraction time and providing a mental challenge); 5) provision of vegetation, bones, ice blocks with food, whole food or carcasses, etc. (i.e. increase the time to process and ingest food); 6) increase of dietary fibre content (i.e. promote satiety); 7) feeding at irregular time intervals (i.e. decrease the predictability of feeding times); or a combination of two or more of the aforementioned options (Shepherdson et al., 1993; Brouns et al., 1994; Young et al., 1994; Newberry, 1995; Bauck, 1998; Aerni et al., 2000; Young, 2003; Hernandez-Divers, 2006; Honess and Marin, 2006; van Krimpen et al., 2009). The applicability of the various approaches, however, may vary across species, depending on species-specific characteristics such as the animals' intelligence, natural diet, methods of food procurement and foraging pattern. In parrots, the following approaches have been applied: 1) provision of food in multiple locations (e.g. Coulton et al., 1997; van Hoek and King, 1997); 2) provision of food hidden in the enclosure and/or scattered in between inedible materials (e.g. van Hoek and King, 1997; Elson and Marples, 2001); 3) provision of foraging devices or puzzle feeders (e.g., Coulton et al., 1997; Bauck, 1998; Meehan et al., 2002; Lumeij and Hommers, 2008); and 4) provision of larger-sized pellets (Rozek and Millam, 2011). The relative merits of each of the individual approaches promoting foraging behaviour are, however, unclear because they have usually been applied as a combination, confounded with nutritional content, tested on a limited number of animals, and/or tested sequentially, leading to potential bias and confounding by residual effects of previous treatments (Newberry, 1995). In addition, comparing the results of different studies is difficult due to differences in study design, thereby emphasizing the need for studies aiming to determine the (differential) effects of the different enrichment approaches that can be used in a specific species. 
To quantitatively evaluate the effects of the different types of foraging enrichment, we conducted a study in Grey parrots (Psittacus erithacus erithacus) in which we compared eleven different foraging enrichments belonging to one of the four enrichment categories previously described. Since a great variety of puzzle feeders is nowadays available, which differ greatly in design and mode of action, potentially exerting a different effect on foraging behaviour and time, no single device was considered fully representative for the category of enrichments aimed at increasing extraction time. Multiple devices, each requiring a different action to extract the food, were therefore tested.

Compared to other types of enrichment, which may lose their enriching effects quickly, foraging devices such as puzzle feeders tend to be more challenging and remain stimulating to the animal, particularly when containing the animal's total daily diet (Shyne, 2006). It was therefore hypothesized that, although all types of foraging enrichments would result in significant increases of foraging times compared to baseline values, differences would be present between the different categories, with puzzle feeders resulting in the highest increases. In addition to establishing (increases in) total foraging times of the different foraging enrichments, we also determined the relative proportions of time spent on the different behaviours (i.e., searching, procuring and consumption of food) as each enrichment is intended to influence foraging behaviour in its own way (e.g. the use of multiple food bowls will mainly increase time spent on locomotory behaviours as an animal needs to move from one location to the other, whereas offering larger-sized particles of food will mainly affect time spent on consumption of food). The choice for a specific enrichment may therefore not only depend on its efficacy to increase foraging time, but also on the type of activity promoted by it, which preferably resembles the natural foraging activity of the species studied. Other factors that may affect the choice for a specific type of foraging enrichment include the time that is needed for the animal to learn how to use the enrichment (i.e. the item preferably has a steep learning curve) and the presence of a habituation effect (which is preferably non-existent, as this may render the enrichment less effective over time). We therefore also included these parameters in our study and monitored both the time span needed by the parrots to master the foraging technique and be able to obtain its daily food intake from the enrichment (i.e. the learning curve) and the presence of a decrease in the amount of foraging over a one-week period, which is the shortest time interval during which a habituation effect has previously been noted in other species (Kuczaj et al., 2002). Results of this study will help make evidence-based decisions on the best way to provide foraging enrichment to (Grey) parrots to meet their welfare needs.

\section{Materials and methods}

\subsection{Ethical approval}

Prior to performing the study ethical approval was obtained from the Institutional Animal Care and
Use Committee of Utrecht University (DEC 2011. I.02. 018).

\subsection{Animals}

In this study, twelve Grey parrots (Psittacus erithacus erithacus) were used, of which six females and six males. Ages of the birds ranged from 6 months to 6 years. Ten of the parrots were parent-raised birds that had experienced minimal contact with humans prior to fledging. The two remaining birds (one male, one female) were separated from their parents as chicks and hand-raised with siblings. After fledging, all except one bird had near-daily contact with both humans and other Grey parrots. The other parrot (male) was housed individually in a private home until the age of 5 years, after which it was added to our research flock. Here, the parrots were group-housed in groups of six birds. All birds had been exposed to enrichment (e.g., branches, ropes, toys) prior to the study, but none of the birds had prior experience with the use of foraging enrichment.

Prior to participating in the study, the parrots were examined and deemed healthy on the basis of a physical and faecal examination, and tested negative for Psittacine Beak and Feather Disease, Avian Bornavirus, Polyomavirus, and Chlamydia psittaci. Birds showed no signs of feather damaging behaviour or other behavioural disorders. Throughout the study, the parrots' health and overall condition was ensured by daily assessment of their mentation (i.e. overall attentiveness and reaction to environmental cues), activity level, appetite and appearance of the faeces. In addition, physical examinations and monitoring of the weight was performed on a weekly basis.

\subsection{Housing and nutrition}

During the study, parrots were housed individually in standard parrot cages $(L \times W \times H=80 \mathrm{~cm} \times$ $60 \mathrm{~cm} \times 155 \mathrm{~cm})$. Each cage was equipped with two (wooden or rope) perches, a water- and food bowl which were located on the ground-level. All enclosures were located in the same room, with visual barriers installed between the cages to prevent the parrots from synchronizing or copying their behaviour. Vocal contact remained possible throughout the study to prevent complete isolation. The room in which the parrots were housed was lit by natural daylight. In addition, artificial lighting was present according to a 10:14 h light:dark schedule, with lights turned on at 8:00 h.

Prior to commencing the study, birds were allowed to acclimatize to their novel environment for a period of two weeks. During this period, food was supplied in a regular food bowl. All parrots were fed an extruded diet (Scenic paradise mix, Plymouth, USA; energy content $\sim 2 \mathrm{kcal} / \mathrm{g}$ ), of which they received 60 grams per day ( $\sim$ daily food consumption). Food consumption of the parrots was monitored on a daily basis to ensure sufficient food intake by the parrots. Fresh drinking water was available ad libitum. Refilling of the food bowls or foraging enrichments and cleaning of the cages occurred daily between 9:00 and $10: 00 \mathrm{~h}$. 
(a)

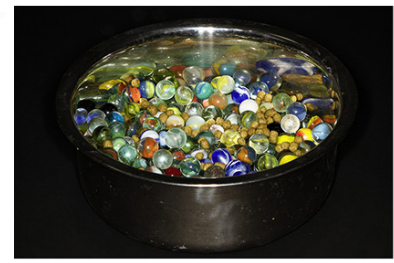

(b)

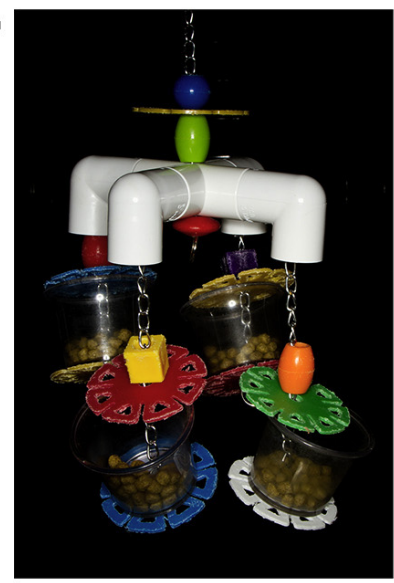

(h)

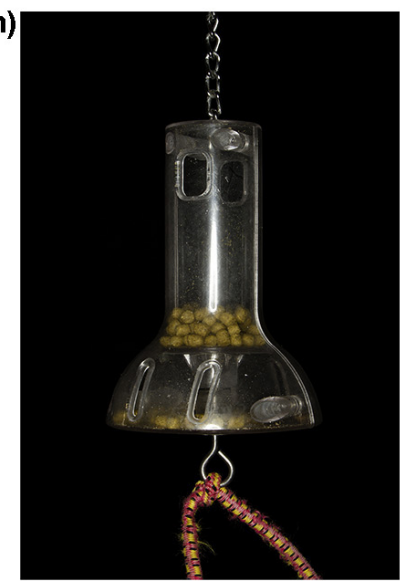

(c)

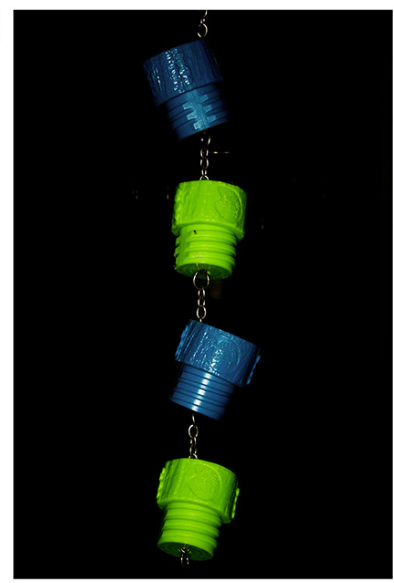

(d)

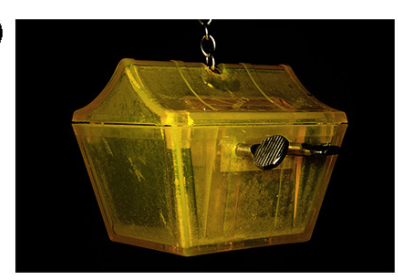

(i)

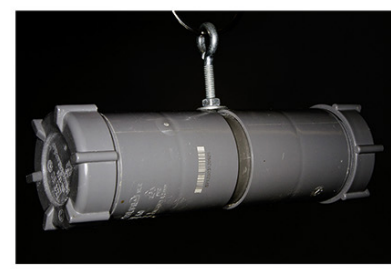

(j)

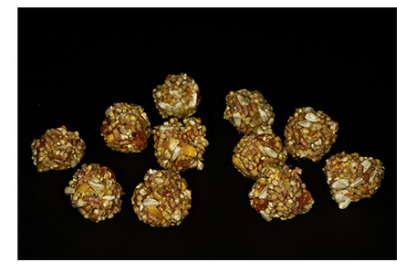

(e)

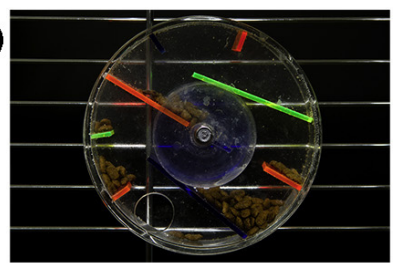

(f)

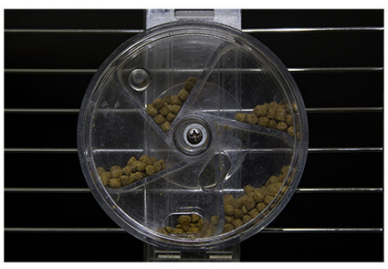

(g)

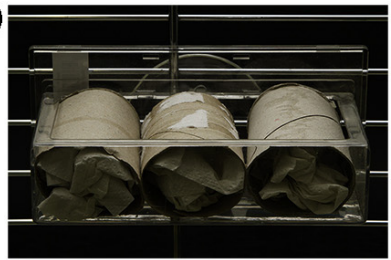

Fig. 1. a-j Different enrichments used in the study (see Table 1 for further explanation).

\subsection{Enrichments}

Eleven different foraging enrichments were tested in this study (Fig. 1a-j). Descriptions and abbreviations are given in Table 1 . These enrichments could be divided into one of the following four enrichment approaches: 1) increasing the spatial distribution of food in the enclosure by providing the pellets in multiple food bowls rather than one location; 2) increasing search time by mixing the pellets with inedible items; 3 ) increasing extraction time by offering the pellets in foraging devices or puzzle feeders; and 4) increasing the time needed to process and ingest food by providing larger-sized food particles. As there is a huge variety in commercially available puzzle feeders, each exerting a different effect on foraging behaviour and time, we selected eight different devices for testing purposes based on the following two criteria: a) the device should be able to contain the daily amount of food; and $b$ ) the parrot has to perform an action (e.g., lift, spin, shake, unscrew, pull, shred, pick particles out of a hole) that was different for each puzzle feeder tested. When food was mixed with inedible material (ST1), marbles instead of bedding materials were chosen as the latter have been reported to cause gastrointestinal obstructions or impactions if ingested by the parrot (e.g., Cannon, 1992; Lumeij, 1994; Morissey, 1996). The marbles (diameter $1.5 \mathrm{~cm}$ ) were considered to be large enough not to be swallowed by the parrot, but small enough to be manipulated with the beak. For the enrichment aimed at increasing processing time (PT1), a different type of food had to be used compared to the food used for the other types of enrichment as the latter pellets were only available in a regular size (approximately $8 \mathrm{~mm}$ diameter) and not in the size desired for this particular enrichment (i.e., $2.5 \mathrm{~cm}$ diameter).

\subsection{Study design}

In the first four days following the acclimatization phase, video recordings were made of the individual parrots to establish baseline foraging times (i.e., foraging from a regular food bowl). 
Table 1

Descriptions of the foraging enrichments ${ }^{\mathrm{a}}$ used in the experiment.

\begin{tabular}{|c|c|c|}
\hline $\begin{array}{l}\text { Abbreviations of } \\
\text { enrichment }\end{array}$ & Description & Strategy \\
\hline SD1 & $\begin{array}{l}\text { Multiple (4) regular-sized food bowls, each containing } 15 \text { grams of pellets, of } \\
\text { which two were hung at the level of the highest perch and two were placed on } \\
\text { ground-level, on opposite sides of the cage. }\end{array}$ & $\begin{array}{l}\text { Increase spatial distribution of } \\
\text { food }\end{array}$ \\
\hline ST1 & $\begin{array}{l}\text { Large stainless steel pet food bowl }(2.8 \mathrm{~L} \text {, diameter } 24 \mathrm{~cm} \text { ) filled with } \\
\text { coloured-glass marbles (diameter } 1.5 \mathrm{~cm} \text { ) and pellets mixed together (Fig. 1a). } \\
\text { Placed on ground level. }\end{array}$ & $\begin{array}{l}\text { Increase search time by mixing } \\
\text { with inedible items }\end{array}$ \\
\hline ET1 & $\begin{array}{l}\text { Foraging device consisting of four refillable plastic transparent cups covered } \\
\text { with a lid and dangling from a PVC pipe (Fig. 1b). Can be hung from the ceiling. }\end{array}$ & Increase extraction time \\
\hline ET2 & $\begin{array}{l}\text { Plastic foraging device consisting of four opaque cups that can be hung from } \\
\text { the ceiling and need to be unscrewed (Fig. 1c). Initially the cups can be } \\
\text { tightened slightly after placing food in it, but once parrots get more } \\
\text { experienced with unscrewing the cups, they can be tightened faster. }\end{array}$ & Increase extraction time \\
\hline ET3 & $\begin{array}{l}\text { Transparent acrylic treasure chest with two stainless-steel keys that can be } \\
\text { used to lock the chest once it has been filled with food (Fig. 1d). Parrots need } \\
\text { to turn and pull the keys to unlock the box, after which the bottom drops to } \\
\text { reveal the food. For an easier skill level only one key can be locked. }\end{array}$ & Increase extraction time \\
\hline ET4 & $\begin{array}{l}\text { Transparent acrylic wheel (diameter } 15 \mathrm{~cm} \text { ), with a maze present inside } \\
\text { (Fig. 1e). Parrots need to spin and turn the wheel in order to gain access to food } \\
\text { via a large circular hole (diameter } 2.5 \mathrm{~cm} \text { ) in the front. }\end{array}$ & Increase extraction time \\
\hline ET5 & $\begin{array}{l}\text { Transparent acrylic wheel (diameter } 15 \mathrm{~cm} \text { ) with } 6 \text { chambers which can be } \\
\text { filled with food and hung to the side of the cage (Fig. } 1 \mathrm{f} \text { ). Birds can turn the } \\
\text { front of the wheel to access a specific chamber via two oval-shaped openings } \\
\text { (size } 1.5 \times 2.5 \mathrm{~cm} \text { ) in the front. }\end{array}$ & Increase extraction time \\
\hline ET6 & $\begin{array}{l}\text { Honey-comb transparent acrylic feeder }(W \times L=8 \times 18 \mathrm{~cm}) \text { in which a } \\
\text { cardboard box filled with food can be placed (Fig. } 1 \mathrm{~g}) \text {. To access food, birds } \\
\text { have to shred the cardboard. }\end{array}$ & Increase extraction time \\
\hline ET7 & $\begin{array}{l}\text { Transparent acrylic capsule (height } 15 \mathrm{~cm} \text {, diameter } 10 \mathrm{~cm} \text { ) in which food can } \\
\text { be placed (Fig. } 1 \mathrm{~h} \text { ). Parrots need to pull down a platform in order to have } \\
\text { pellets drop in the lower compartment which can then be accessed via holes in } \\
\text { the side. }\end{array}$ & Increase extraction time \\
\hline ET8 & $\begin{array}{l}\text { Opaque PVC tube with two holes }(9 \mathrm{~mm}) \text { drilled in it(Fig. } 1 \mathrm{i}) \text {. After placing } \\
\text { pellets inside the toy can be hung from the ceiling. Pellets can be removed by } \\
\text { swinging the toy or manipulating pellets out of the hole with the beak (Lumeij } \\
\text { and Hommers, 2008). }\end{array}$ & Increase extraction time \\
\hline PT1 & $\begin{array}{l}\text { Nutritionally balanced mix of savoury peanuts, hulled canary seed, cracked } \\
\text { corn and pellets, shaped into the form of a berry (diameter } 2.5 \mathrm{~cm} \text {; Fig. } 1 \mathrm{j} \text { ). } \\
\text { Provided into a regular food bowl placed on ground-level. They weigh } \\
\text { approximately } 4 \text { grams each and contain } 12.5 \mathrm{kcal}(\sim 3 \mathrm{kcal} / \mathrm{g}) \text {. }\end{array}$ & $\begin{array}{l}\text { Increase time needed to } \\
\text { process and ingest food by } \\
\text { providing larger-sized food } \\
\text { particles }\end{array}$ \\
\hline
\end{tabular}

a Names and manufacturers of commercially available enrichments (ET1 - ET7; PT1) are available upon request.

Next, foraging enrichments were introduced to the parrots. The order in which the items were presented differed for each parrot and was determined at random using a random sequence generator programme (http://www.random.org/).

For the foraging enrichment aimed at increasing spatial distribution of food (SD1), increasing search time (ST1) and increasing process time (PT1) no acclimatization period was considered necessary and all food was immediately placed in the enrichment. For the foraging enrichments aimed at increasing extraction time (ET1-ET8) an acclimatization period was present, during which the devices were gradually introduced to the birds to prevent provocation of a fear response and/or onset of anorexia due to sudden exposure and unfamiliarity with the novel item. When the novel item did not elicit a fear response, it was immediately hung or placed in the cage. In the rare occasion that a fear response was elicited, the item was hung outside the cage and placed inside as soon as the parrot did not respond fearful anymore (which took approximately 2-3 days).

After two days of acclimatization to the novel foraging enrichment (during which food was presented in a regular food bowl and the empty device was placed in the cage), the daily food consumption was presented in the enrichment, with an additional one-third provided in the food bowl. If a parrot had eaten between 50 and $90 \%$ of its daily food consumption (i.e. at least $17 \%$ from the device), no changes were made to the protocol until the parrots food intake increased to $>90 \%$ of baseline values (i.e. the parrot was able to obtain at least $57 \%$ of its food intake from the device). Once the parrot had consumed $>90 \%$ of its daily food intake, the next day food would be supplied in the enrichment only, without additional food present in the food bowl.

If a parrot had consumed less than $50 \%$ of its daily food intake, the next day the amount of food supplied in the food bowl was increased to $75 \%$ of the daily food intake and remained at $75 \%$, until the parrot's food intake increased to $>90 \%$ of baseline values (i.e. the parrot was able to obtain at least $15 \%$ of its food intake from the device). Subsequently, the amount of food in the food bowl would be decreased with 10 grams per day until the total daily food consumption was obtained via the device.

For toys with a varying difficulty level (ET2, ET3, ET7) the enrichment device was initially tried at the lowest difficulty level, and upgraded to the highest difficulty level when the parrots were able to consume their daily food consumption 
Table 2

Ethogram of foraging related behaviours displayed by Grey parrots.

\begin{tabular}{|c|c|}
\hline Behavioural categories and underlying behaviours & Description \\
\hline \multicolumn{2}{|c|}{ Locomotory behaviours ${ }^{\mathrm{a}}$ (movement towards a feeding site) } \\
\hline Sidle & Moving sideways by sliding the feet one by one along the perch \\
\hline Walk & Moving by placing one foot in front of the other \\
\hline Climb & Moving upwards along the bars of the cage (assisted with the beak) \\
\hline Descend & Moving downwards along the bars of the cage (assisted with the beak) \\
\hline \multicolumn{2}{|c|}{ Enrichment-related behaviours (manipulation of enrichment item) } \\
\hline Pick up item & Pick up enrichment item \\
\hline Carry item & Walking with the enrichment item in the beak \\
\hline Drop item & Dropping enrichment item \\
\hline Manipulate enrichment & Using beak and/or paws to manipulate the foraging enrichment \\
\hline Podo-mandibulation item & Holding enrichment item in the foot, while manipulating it with the beak \\
\hline \multicolumn{2}{|c|}{ Food-related behaviours (food procurement and consumption) } \\
\hline Search food & Search for food in bowl or on the ground \\
\hline Pick up food & Pick up food \\
\hline Carry food & Walking with the food item in the beak \\
\hline Drop food & Dropping food on the ground or in the water bowl \\
\hline Podo-mandibulation food & Holding food in the foot, while manipulating it with the beak \\
\hline Feed from bowl & Consuming food directly from the bowl with the beak \\
\hline Feed from enrichment & Consuming food directly from the foraging enrichment with the beak \\
\hline Feed from ground & Consuming food directly from the ground with the beak \\
\hline Feed from foot & Consuming food while holding it in the foot \\
\hline Drink & Taking and swallowing water with beak \\
\hline Soak & Placing food in the water prior to consuming it \\
\hline
\end{tabular}

a Only included when the locomotory action is directly followed or preceded by an enrichment- or food-related behaviour.

from the device. Upon increasing the difficulty level, parrots were given 10 grams $(\sim 17 \%)$ of food in a food bowl to prevent starvation, until they were able to consume at least $70-80 \%$ of food from the device, at which time the difficulty level would be increased again (if applicable). Records were kept of the time that the parrots needed to master the use of the foraging enrichment (i.e., the time at which the parrot was able to obtain all the food from the enrichment, starting from the moment that food was first offered via this enrichment). These data were subsequently used to determine learning curves and assess overall difficulty level of the toy.

Once parrots were able to obtain their daily food intake from the enrichment device, the use of the device was evaluated for a period of seven days. Subsequently, this device was removed from the cage and, starting the next day, a new enrichment was gradually introduced according to the protocol described previously.

When a parrot was not able to use the enrichment (in its highest difficulty level) to obtain its daily food requirement within a period of three weeks and showed no further progress, the training with this device was temporarily ceased and a second attempt was made after the bird had finished the experiment with the remaining enrichments. Once the parrot had learned to use the device, the durations of the first and second attempt were combined to determine the total learning time. If this attempt was again unsuccessful after a period of 4-5 weeks, the use of the foraging enrichment was qualified as 'failed'.

\subsection{Behavioural analyses}

Throughout the study, video recordings were made of the parrots' behaviour during the day time. This prevented influence of observer presence on the parrots' behaviour while at the same time allowing the total foraging time and the proportion of time spent on the parrots' foraging activities (Table 2) to be determined at a later time.

Total foraging times were scored as the total amount of time spent on foraging-related movement (walking, sidling, climbing), enrichment-related behaviours (manipulating the enrichment) and food-related behaviours (search, procurement and consumption of the food). Feeding furthermore included drinking or soaking pellets in water prior to eating them.

In addition to scoring the total foraging times and relative time spent on the different foraging-related activities, lengths, frequencies and times of the foraging bouts (i.e. time period from the first contact with the food source until the consumption of the last piece of food in a single, uninterrupted session) were also recorded. Foraging times and bouts were initially scored in seconds, and subsequently rounded to the nearest minute for further analyses. For each enrichment, data of the individual time points (foraging times, food consumption, foraging bouts and frequencies) were averaged to determine the mean values for each parrot.

\subsection{Statistical analysis}

SPSS Statistics Software version 20.0 (IBM, Armonk, USA) was used for the statistical analysis. Data were expressed as means $\pm \mathrm{SD}$, unless stated otherwise. $P$ values $<0.05$ were considered statistically significant unless stated otherwise.

To test whether the various foraging enrichments resulted in a significant increase in foraging times compared to the baseline values, a linear mixed-effects model (LME) was used. In this model, the daily foraging data for each individual parrot were entered as separate data 
points, with parrots included as between-subjects variable and days as repeated measurements. In addition to our variable of interest (i.e. type of foraging enrichment), age, gender, rearing method and day were included in the initial model as potential explanatory variables in order to exclude these factors from biasing the results. Prior to interpreting the results, assumptions of the linear mixedeffect model (linearity of the results, normality of residuals, random intercepts and slopes, independence of the residuals) were checked. A similar model was also used to test whether significant differences were present in food consumption, foraging bouts and frequencies with the different foraging enrichments (compared to baseline).

To determine which foraging-related activities (locomotory, enrichment-related and/or food-related behaviours) led to increases in foraging times for each of the enrichments, actual time spent on these activities were compared with baseline values. In addition, relative time spent on the different activities was compared between the different enrichments to determine whether the proportions of time spent on the different activities would differ significantly. For these purposes repeated measures ANOVAs were performed if data were normally distributed (or became normally distributed with an arcsine transformation) and Mauchley's test of sphericity demonstrated homogeneity of variance for the parameters. If transformations did not result in a normal distribution of the data, a Friedman test was used for comparison of the enrichments (including baseline for absolute times; excluding baseline for relative times). If data was missing for one or more parrots, separate analyses were performed (ET3 $[N=6]$, SD1 $[N=9]$ ).

To assess the mean $( \pm S D)$ time needed for a parrot to master the use of the foraging enrichment, a Kaplan-Meier analysis was performed. If a second attempt was needed, lengths of the first and second period were combined. Whenever a parrot was unable to master the use of the toy, the attempt was labelled as a censored event at the number of days that the enrichment was offered to the parrot. In addition to the Kaplan-Meier analysis, a Log rank Mantel-Cox test was performed to analyze whether differences were present in the 'learning curves' for the various enrichments.

When the previous models revealed significant differences for the foraging enrichments (including baseline), post hoc tests were performed, in which comparisons were made between the average values of the individual foraging enrichments and baseline values. For this purpose, one- or two-sided paired $t$-tests were used if the Kolmogorov-Smirnoff test demonstrated a normal distribution of the (transformed) data. If normal distribution could not be achieved, non-parametric tests were used. To account for multiple testing, a Bonferroni correction ( $\alpha /$ number of comparisons) was applied, which resulted in a corrected $\alpha$ of $0.0045(=0.05 / 11)$. Post hoc comparisons were also performed to determine statistically significant differences between the different foraging enrichments, in which the most-effective enrichment (i.e. enrichment resulting in the highest average foraging time) was compared with bottom-three enrichments (i.e. the least effective enrichments, resulting in the lowest average foraging time) and vice versa. For these results, a Bonferroni correction resulted in an adjusted $P$-value of 0.0167 $(=0.05 / 3)$.

To test whether a learning or habituation effect was present, a repeated measures ANOVA was performed using a polynomial contrast analysis. This analysis was performed for each of the enrichments separately and aimed specifically to identify a downwards linear or quadratic trend. As two separate tests had to be performed to assess the presence of one of these trends, a Bonferroni post hoc correction was applied, resulting in a corrected significance level $(\alpha)$ of $0.025(=0.05 / 2)$. Upon finding a downwards linear or quadratic trend for a specific enrichments, pairwise comparisons were made between the data of the day 1 and 7 with a one-sided paired $t$-test to determine whether the average foraging times at last day (day 7) were indeed significantly lower than the foraging times at the first day (day 1).

\section{Results}

Throughout the study, all parrots remained in good health and were found to be alert, active and responsive to their environment. The parrots were able to maintain a stable body weight, with minor week-to-week changes. One of the parrots developed a mild form of feather damaging behaviour shortly after being introduced into the study. A lack of social contact was suspected as underlying cause. As none of the birds received the same foraging enrichment simultaneously, we decided to remove the visual barrier between the cages for this one bird to allow visual contact with one of its neighbours. This measure was successful as the feather damaging behaviour ceased almost instantaneously and it was therefore decided not to remove the bird from the study.

\subsection{Baseline foraging activity and food consumption}

Baseline foraging times and food consumption were found to be relatively similar for most parrots, with only minor day-to-day fluctuations present. During the analysis of the video recordings, one parrot (female, 1 year) was, however, identified as an outlier, as her baseline foraging times were approximately five-fold higher than those of the other parrots $(247 \pm 66 \mathrm{~min}$ /day versus $47 \pm 18 \mathrm{~min} /$ day). Analysis of this parrot's behaviour identified that she, in contrast to the other parrots, did not climb down to the food bowl and remained seated there during a foraging bout, but rather climbed up and down every single time to collect one or a few pellets and carry these up to eat them while seated at the highest perch. As this behaviour pattern was found to be distinctly different from that of the other parrots, this parrot was excluded from further analysis of the foraging activities. Data analysis of the remaining 11 parrots subsequently revealed that the parrots spent $47 \pm 18$ min per day on foraging-related activities during which they consumed approximately $44 \pm 9 \mathrm{~g}$ of pellets. Feeding bouts occurred approximately $17 \pm 9$ times per day, with each bout lasting approximately $3.1 \pm 1.4$ minutes. Feeding bouts typically 
took place before $11: 00 \mathrm{~h}$ or after $14: 00 \mathrm{~h}$, with reduced activity during midday.

\subsection{Time needed to master the use of the different foraging enrichments (learning curves)}

Most parrots were able to master the use of a foraging enrichment within the first attempt. A second attempt was, however, needed for five of the puzzle feeders as one or more of the parrots were not able to utilize the devices within three weeks. The devices that needed a second attempt included ET1 $(N=1)$, ET2 $(N=1)$, ET3 $(N=8)$, ET7 $(N=1)$, and ET8 $(N=2)$. In most cases, the birds were usually successful in their second attempt to master the foraging technique. For ET3, however, the training period was insufficient to teach the parrots how to use the device $(N=6)$.

It took $8.3 \pm 1.1$ days (starting from the initial presentation of food in the enrichments) before the parrots were able to master the technique and consume their total daily intake from the enrichments. Considerable differences were, however, present between the learning curves for the different enrichments (Mantel-Cox rank test, $\chi^{2}=91.654 ; \mathrm{df}=10 ; P<0.001$; Table 3). Pairwise comparisons revealed that significantly more time was needed for the parrots to master the technique needed to open the ET2 (12.7 \pm 1.6 days; $P<0.004$ for all enrichments except the ET7 and ET8) and the ET3 (39.1 \pm 6.6 days; $P<0.001$ for all enrichments; Table 3). In contrast, the period needed for the parrots to learn how to obtain food from the SD1

Table 3

Number of days needed for the parrots $(N=12)$ to learn to be able to use the various types of foraging enrichment. A value of 0 indicates that birds did not need time to master the foraging technique, but were able to employ it directly on the first day of presentation.

\begin{tabular}{|c|c|c|}
\hline Enrichment & Mean \pm SD & Range (min-max) \\
\hline $\mathrm{SD} 1^{\mathrm{a}}$ & $1.3 \pm 0.3$ & $0-3$ \\
\hline ST1 & $4.3 \pm 1.0$ & $1-12$ \\
\hline ET1 ${ }^{\mathrm{e}}$ & $5.3 \pm 1.4$ & $0-16$ \\
\hline $\mathrm{ET}^{\mathrm{b}, \mathrm{e}}$ & $12.7 \pm 1.6$ & $6-23$ \\
\hline ET3 $^{c, g}$ & $39.1 \pm 6.5$ & $4->60$ \\
\hline ET4 & $5.3 \pm 0.6$ & $2-8$ \\
\hline ET5 & $2.5 \pm 0.7$ & $1-9$ \\
\hline ET6 & $5.6 \pm 1.5$ & $2-20$ \\
\hline ET7 $^{\mathrm{e}}$ & $6.1 \pm 1.7$ & $2-23$ \\
\hline ET8 $^{\mathrm{f}}$ & $6.6 \pm 2.8$ & $1-28$ \\
\hline PT1 ${ }^{d}$ & $1.8 \pm 0.4$ & $0-4$ \\
\hline Overall & $8.3 \pm 1.1$ & $0->60$ \\
\hline
\end{tabular}

a Log rank Mantel-Cox (pairwise comparisons) demonstrated significant differences $(P<0.0045)$ compared to the use of all other foraging devices except the ET1 $(P=0.006)$, ET5 $(P=0.071)$, ET8 $(P=0.011)$ and PT1 $(P=0.190)$.

b Log rank Mantel-Cox (pairwise comparisons) demonstrated significant differences $(P<0.0045)$ compared to the use of all other foraging enrichments except the ET7 and ET8.

c Log rank Mantel-Cox (pairwise comparisons) demonstrated significant differences $(P<0.001)$ compared to the use of other foraging enrichments.

d Log rank Mantel-Cox (pairwise comparisons) demonstrated significant differences $(P<0.001)$ compared to the ET2, ET3, ET4 and ET7.

e Had to be presented for a second time $(N=1)$.

f Had to be presented for a second time $(N=2)$.

g Had to be presented for a second time $(N=8) ; 6$ parrots were unable to use the device within the time it was offered to them.
( $1.3 \pm 0.3$ days $)$ and PT1 ( $1.8 \pm 0.4$ days $)$ was significantly shorter (Table 3).

For some of the enrichments, the time needed to master a specific foraging technique varied considerably between the different parrots, ranging from a few days to more than two weeks. No differences were, however, detected when comparing the learning curves for the different parrots $\left(\chi^{2}=16.249 ; \mathrm{df}=11 ; P=0.132\right)$, indicating that none of the parrots could consistently be identified as a faster or slower learners than the other parrots across the range of enrichments tested.

\subsection{Effects of foraging enrichments on foraging times, foraging bouts and food consumption}

Throughout the study, day-to-day fluctuations were present in the daily food consumption of the individual parrots. Foraging enrichments affected food consumption significantly (LME; $F_{11,510}=26.959 ; P<0.001$; Table 4 ). In general, slight to moderate increases in food consumption were seen, with exception of the ST1 from which the parrots appeared to consume less food. Pairwise comparisons, however, only demonstrated significant differences compared to baseline for the ET8 (paired $t$-test: $t=-4,263$; $\mathrm{df}=10 ; P=0.002$ ) and PT1 (paired $t$-test: $t=-5,213 ; \mathrm{df}=10$; $P<0.001$ ).

Upon analysis of the foraging times for SD1, two parrots were found to consistently throw the higher placed food bowls on the floor of the cage, after which they would spend a considerable time foraging on the ground. As this unintended behaviour doubled foraging times, actual foraging activity could not be reliably evaluated. The two birds were therefore removed from further analyses of SD1.

All foraging enrichments were successful in increasing the mean foraging times of parrots compared to baseline (Fig. 2). Average foraging times ranged from $68 \pm 27 \mathrm{~min}$ for the least-effective foraging enrichment (i.e., SD1) to $123 \pm 52$ minutes for the most-effective foraging enrichment (i.e., ET6; Table 4). The type of enrichment had a significant effect on foraging times $\left(\right.$ LME; $\left.F_{11,497}=15.296 ; P<0.001\right)$. In contrast, gender (LME; $F_{1,7}=0.247 ; P=0.634$ ), age (LME; $F_{1,8}=2.983 ; P=0.124$ ), rearing-method (LME; $F_{1,7}=0.063 ; P=0.809$ ) and day (LME; $F_{1,502}=2.460 ; P=0.117$ ) were not found to significantly influence foraging times.

Pairwise comparisons of the foraging enrichments with baseline values revealed nine of the eleven foraging enrichments to result in significantly longer foraging times (one-sided, paired $t$-tests: $P<0.0045$; Table 4). For the ET3 and SD1, no statistically significant differences could be demonstrated compared to baseline (one-sided, paired $t$ tests: $t=3.201 ; \mathrm{df}=5 ; P=0.012$ for ET3; $t=2.568 ; \mathrm{df}=8$; $P=0.017$ for SD 1 ). A post hoc power analysis of the available data revealed that a minimum of 29 and 31 parrots would be needed to demonstrate a statistical significant increase in foraging time $(P<0.045$, power $=0.80)$ for ET3 and SD1, respectively.

Pairwise comparison of the most effective enrichment (ET6) with the bottom-three (SD1, ET3, ET5) revealed significant differences between foraging times that were achieved with the enrichments $(P<0.001 ; P=0.012$ and 
Table 4

Average food consumption, foraging times, bout lengths and frequencies for the parrots $(N=11)$ with the different types of foraging enrichment (mean \pm SD).

\begin{tabular}{|c|c|c|c|c|}
\hline Enrichment & $\begin{array}{l}\text { Food consumption } \\
\text { (g/day) }\end{array}$ & $\begin{array}{l}\text { Foraging time } \\
\text { (min/day) }\end{array}$ & $\begin{array}{l}\text { Average bout } \\
\text { length (min) }\end{array}$ & $\begin{array}{l}\text { Average bout } \\
\text { frequency (\#) }\end{array}$ \\
\hline Baseline (food bowl) & $44 \pm 9$ & $47 \pm 18$ & $3.1 \pm 1.4$ & $17 \pm 9$ \\
\hline $\mathrm{SD} 1(N=9)$ & $49 \pm 8$ & $68 \pm 27^{\mathrm{d}, e}$ & $4.0 \pm 2.2$ & $23 \pm 7$ \\
\hline ST1 & $37 \pm 5$ & $95 \pm 32^{c}$ & $6.7 \pm 2.5^{\mathrm{b}}$ & $15 \pm 5$ \\
\hline ET1 & $51 \pm 7$ & $100 \pm 54^{d}$ & $4.8 \pm 1.4$ & $21 \pm 8$ \\
\hline ET2 & $49 \pm 7$ & $116 \pm 38^{c, g}$ & $5.9 \pm 1.7^{\mathrm{b}}$ & $21 \pm 6$ \\
\hline ET3 $(N=6)$ & $50 \pm 7$ & $76 \pm 39^{f}$ & $4.3 \pm 1.3$ & $17 \pm 7$ \\
\hline ET4 & $51 \pm 7$ & $85 \pm 38^{d}$ & $5.0 \pm 1.4^{\mathrm{b}}$ & $17 \pm 7$ \\
\hline ET5 & $47 \pm 8$ & $81 \pm 36^{\mathrm{d}, \mathrm{e}}$ & $6.6 \pm 4.9$ & $16 \pm 4$ \\
\hline ET6 & $50 \pm 5$ & $123 \pm 52^{\mathrm{c}, \mathrm{g}}$ & $6.2 \pm 2.8$ & $20 \pm 6$ \\
\hline ET7 & $46 \pm 7$ & $113 \pm 51^{\mathrm{c}, \mathrm{h}}$ & $5.2 \pm 1.8^{\mathrm{b}}$ & $22 \pm 7$ \\
\hline ET8 & $53 \pm 5^{b}$ & $108 \pm 42^{c}$ & $5.9 \pm 2.2^{\mathrm{i}}$ & $20 \pm 10$ \\
\hline PT1 & $62 \pm 9^{a}$ & $105 \pm 47^{d}$ & $10.0 \pm 5.5^{b}$ & $11 \pm 4$ \\
\hline
\end{tabular}

a Two-sided paired samples $t$-test; $P<0.001$ compared to baseline.

b Two-sided paired samples $t$-test; $P<0.0045$ compared to baseline.

c One-sided paired samples $t$-test; $P<0.001$ compared to baseline.

d One-sided paired samples $t$-test; $P<0.0045$ compared to baseline.

e One-sided paired samples $t$-test; $P<0.001$ compared to most-effective enrichment (ET6).

f One-sided paired samples $t$-test; $P<0.017$ compared to most-effective enrichment (ET6).

$g$ One-sided paired samples $t$-test; $P<0.001$ compared to least-effective enrichment (SD1).

h One-sided paired samples $t$-test; $P<0.017$ compared to least-effective enrichment (SD1).

i Wilcoxon signed-rank test; $P<0.0045$ compared to baseline.

$P<0.001$ respectively). Compared to this least-effective foraging enrichment (SD1), the top-three most-effective enrichments (ET2, ET6, ET7) resulted in significantly longer foraging times $(P<0.001 ; P<0.001$ and $P=0.004$ respectively).

Similar to baseline, foraging bouts predominantly occurred during the early morning and late afternoon. Frequencies and lengths of the foraging bouts were, however, found to be affected by offering foraging enrichments. In general, increases were seen in both the number and duration of foraging bouts displayed by the parrot (Table 4). A linear mixed-effects model indeed revealed influence of the type of enrichment on these parameters $\left(F_{11,384}=8.750\right.$; $P<0.001$ for bout frequency; $F_{11,384}=12.799 ; P<0.001$ for bout length). Pairwise comparisons of bout frequencies, however, did not reveal significant differences compared to baseline values for any of the enrichments. Pairwise comparisons of bout lengths, on the other hand, showed significant differences between baseline and ET2 (paired $t$-test; $t=3.841 ; \mathrm{df}=10 ; P=0.003$ ), ET4 (paired $t$-test; $t=4.951 ; \mathrm{df}=10 ; P=0.001$ ), ET7 (paired $t$-test; $t=3.683 ; \mathrm{df}=10 ; P=0.004$ ), ET8 (Wilcoxon-signed rank

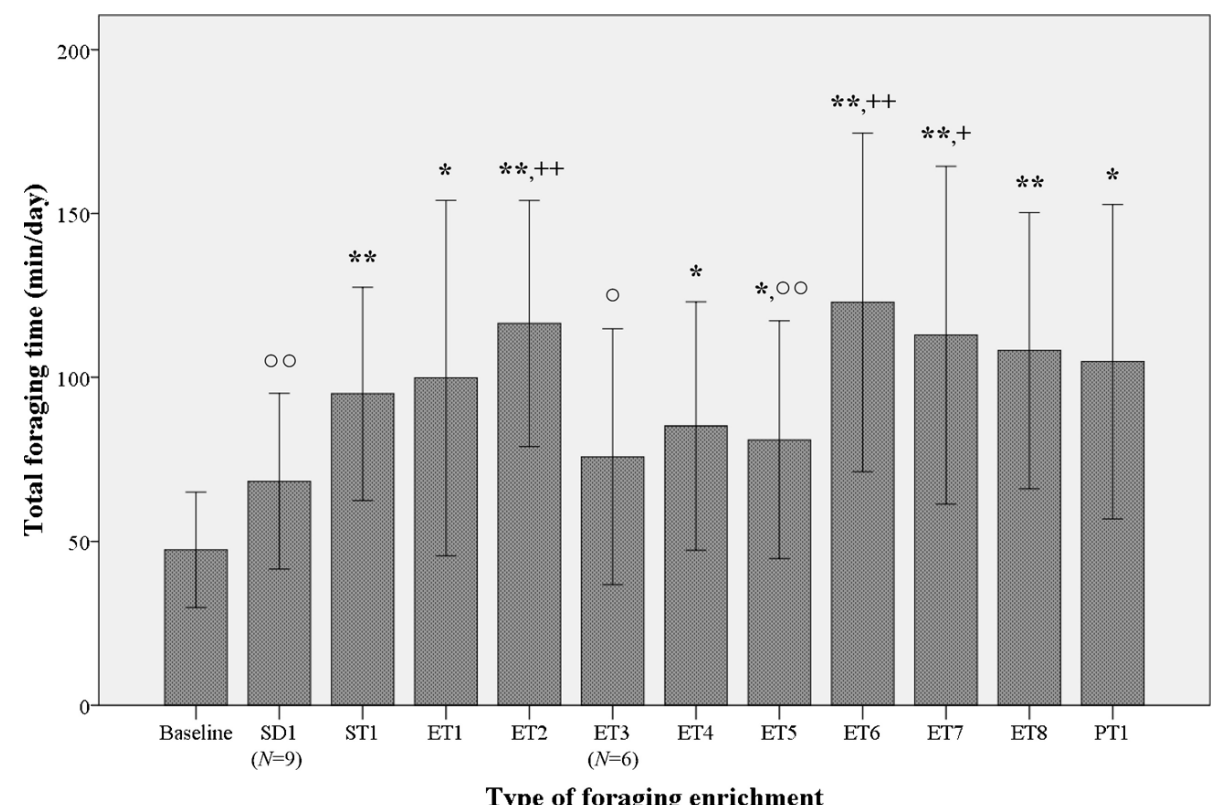

Fig. 2. Foraging times (mean \pm SD) of grey parrots $(N=11)$ using the different foraging enrichments. * Significant difference compared to baseline $(P<0.0045)$. ${ }^{* *}$ Significant difference compared to baseline $(P<0.001)$. ${ }^{+}$Significant difference compared to least-effective enrichment $(P<0.017)$. ${ }^{++}$Significant difference compared to least-effective enrichment $(P<0.001)$. OSignificant difference compared to most-effective enrichment $(P<0.017)$. $\bigcirc \circ$ Significant difference compared to most-effective enrichment $(P<0.001)$. 


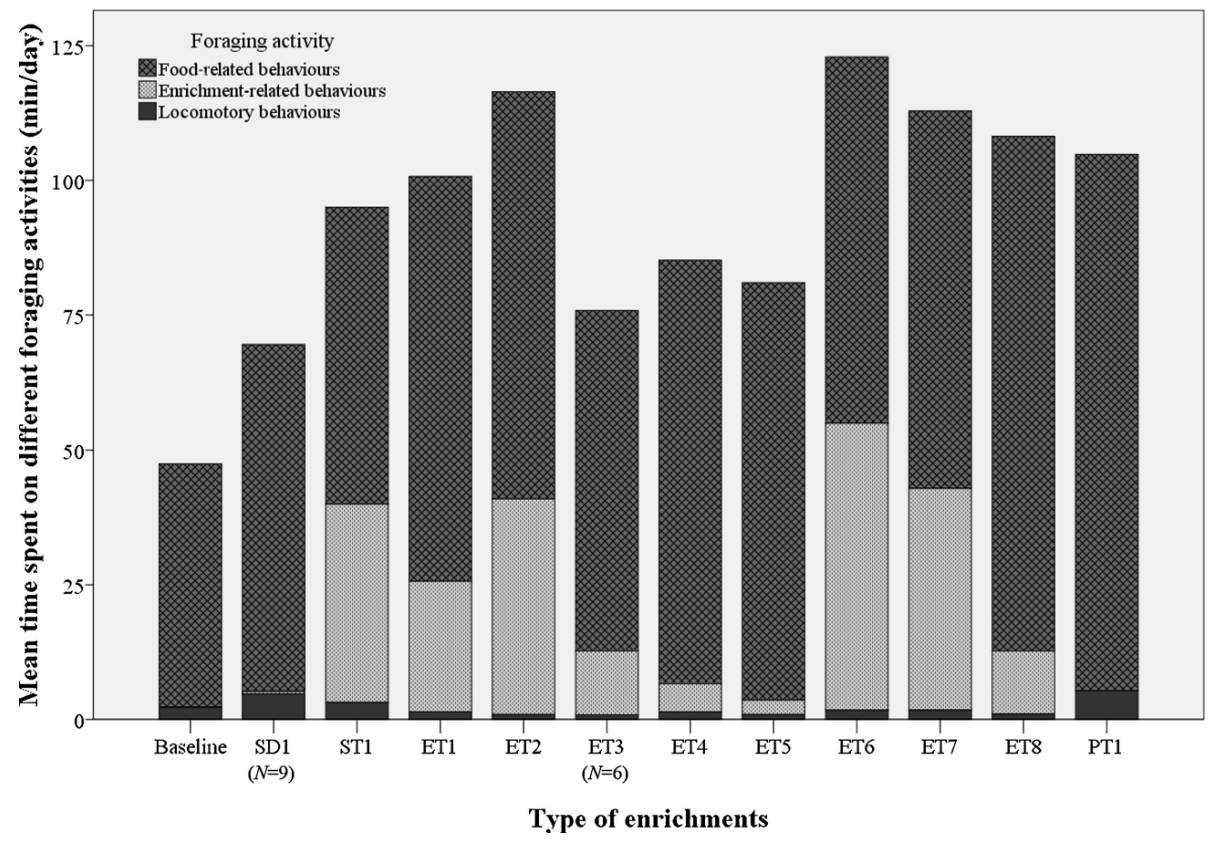

Fig. 3. Relative time distribution spent by parrots $(N=11)$ on the different foraging-related activities using the different foraging enrichments.

test: $Z=2.845 ; N_{1}=N_{2}=11 ; P=0.004$ ), ST1 (paired $t$-test; $t=3.760 ; \mathrm{df}=10 ; P=0.004$ ) and PT1 (paired $t$-test; $t=4.000$; $\mathrm{df}=10 ; P=0.003$ ).

\subsection{Effects of foraging enrichments on time allocation for foraging-related activities}

Absolute and relative times spent on the different foraging related activities were determined for baseline and each of the foraging enrichments (Fig. 3, Table 5). In general, parrots spent little time on movement towards and away from the foraging enrichments (minimum $0.9 \pm 1.0 \mathrm{~min} /$ day $[1 \pm 1 \%]$; maximum $5.4 \pm 4.6 \mathrm{~min} /$ day $[5 \pm 4 \%])$. When comparing the absolute time spent on locomotory behaviours, significant differences were found between the different enrichments (Friedman test: $\chi^{2}=18.642 ; \mathrm{df}=9 ; P=0.034$ ). Significant differences were also present between the foraging enrichments when comparing the proportion of time spent on locomotory behaviours (Friedman test: $\left.\chi^{2}=20.493 ; \mathrm{df}=8 ; P=0.009\right)$. Pairwise comparisons of absolute and relative locomotory times, however, revealed no significant differences with baseline values (Wilcoxon rank tests; $P>0.05$ for absolute times and $P>0.01$ for relative times).

For most devices, the increase in foraging time was mainly a result of time spent on enrichment- and/or foodrelated activities. Significant differences were, however, present between the enrichments in the absolute times spent on interaction with the enrichment (Friedman test: $\left.\chi^{2}=66.125 ; \mathrm{df}=9 ; P<0.001\right)$ and/or interaction with food (Friedman test: $\chi^{2}=27.476 ; \mathrm{df}=9 ; P=0.001$ ). Significant differences were also present between the type of enrichment when comparing the relative time distributions spent on these activities (Friedman rank: $\chi^{2}=34.051$; $\mathrm{df}=7$; $P<0.001$ for foraging activities; Repeated measures ANOVA on arcsin transformed data: $F_{1,10}=1270.154 ; P<0.001$ for feeding activities).

Pairwise comparisons of the absolute times spent on the different activities between foraging enrichments and baseline demonstrated significantly more time spent on

Table 5

Absolute and relative time distribution spent by parrots $(N=11)$ on the different foraging activities with the different types of foraging enrichment.

\begin{tabular}{|c|c|c|c|}
\hline Enrichment & $\begin{array}{l}\text { Locomotor behaviours } \\
\text { min/day (\%) }\end{array}$ & $\begin{array}{l}\text { Enrichment-related } \\
\text { behaviours min/day (\%) }\end{array}$ & $\begin{array}{l}\text { Food-related } \\
\text { behaviours min/day (\%) }\end{array}$ \\
\hline Baseline (food bowl) & $2.3 \pm 2.7(5 \pm 4)$ & 0 & $45 \pm 16(95 \pm 4)$ \\
\hline $\operatorname{SD} 1(N=9)$ & $4.4 \pm 3.5(6 \pm 3)$ & $0.5 \pm 0.6(1 \pm 1)$ & $64 \pm 24(94 \pm 3)$ \\
\hline ST1 & $3.2 \pm 3.0(3 \pm 3)$ & $37 \pm 31^{\mathrm{a}}(36 \pm 24)$ & $55 \pm 21(61 \pm 24)$ \\
\hline ET1 & $1.4 \pm 2.3(1 \pm 2)$ & $24 \pm 31^{\mathrm{a}}(20 \pm 18)$ & $75 \pm 38(79 \pm 17)$ \\
\hline ET2 & $0.9 \pm 1.6(1 \pm 1)$ & $40 \pm 22^{\mathrm{a}}(33 \pm 13)^{\mathrm{b}}$ & $76 \pm 24(66 \pm 14)$ \\
\hline ET3 $(N=6)$ & $0.9 \pm 1.0(1 \pm 1)$ & $16 \pm 16(20 \pm 14)$ & $61 \pm 34(80 \pm 11)$ \\
\hline ET5 & $0.9 \pm 1.6(1 \pm 2)$ & $2.7 \pm 5.1(4 \pm 8)$ & $77 \pm 37(95 \pm 8)^{c}$ \\
\hline ET6 & $1.7 \pm 2.3(1 \pm 2)$ & $53 \pm 35^{\mathrm{a}}(42 \pm 16)^{\mathrm{b}}$ & $68 \pm 30(57 \pm 15)$ \\
\hline ET7 & $1.7 \pm 2.1(2 \pm 2)$ & $41 \pm 50(27 \pm 27)$ & $70 \pm 26(71 \pm 26)$ \\
\hline ET8 & $1.0 \pm 1.3(1 \pm 1)$ & $12 \pm 21(9 \pm 16)$ & $95 \pm 38(90 \pm 15)^{c}$ \\
\hline PT1 & $5.4 \pm 4.6(5 \pm 4)$ & 0 & $99 \pm 46(95 \pm 4)$ \\
\hline
\end{tabular}

a Wilcoxon signed-rank test; $P<0.0045$ compared to baseline.

b Wilcoxon signed-rank test; $P<0.0045$ compared to ET5 (i.e. device with lowest proportion of time spent on foraging).

c Paired $t$-test on arscine transformed data; $P<0.0045$ compared to ET6 (i.e. device with lowest proportion of time spent on feeding). 
foraging for the ST1 (Wilcoxon signed rank test: $Z=-2.803$; $N=11 ; P=0.005)$, ET1 $(Z=-2.934 ; N=11 ; P=0.003)$, ET2 $(Z=-2.934 ; \quad N=11 ; P=0.003)$, ET4 $(Z=-2.934 ; N=11$; $P=0.003)$ and ET6 $(Z=-2.934 ; N=11 ; P=0.003)$. Pairwise comparisons of time spent on feeding activity revealed no significant differences between the different enrichments and baseline (Wilcoxon signed rank tests: $P>0.005$ ).

Of the different puzzle feeders tested, the ET5 resulted in the lowest proportion of time spent on foraging activity $(4 \pm 8 \%)$. Pairwise comparisons of relative time distributions of the remaining enrichments to this device revealed significant higher proportions of time spent on foraging for the ET2 (Wilcoxon signed rank test: $Z=-2.934 ; N=11$; $P=0.003)$ and the ET6 $(Z=-2.934 ; N=11 ; P=0.003)$. The ET6 resulted in the lowest proportion of time spent on feeding activity $(57 \pm 15 \%)$. Pairwise comparisons to the other puzzle feeders identified significantly higher proportions on times spent on feeding from the ET4 $(t=-7.603$; $\mathrm{df}=10 ; P<0.001)$, ET5 $(t=-8.529 ; \mathrm{df}=10 ; P<0.001)$, and ET8 $(t=-7.178 ; \mathrm{df}=10 ; P<0.001)$.

\subsection{Habituation effects to the foraging enrichment over time}

Foraging times of the individual parrots fluctuated on a day-to-day basis over the 7-day observation period. Throughout this period, no obvious decreases in foraging times were identified (Fig. 4). Repeated measures ANOVA with polynomial contrast analysis only revealed a downwards linear or quadratic trend for the ET8 (repeated measures ANOVA: $F_{1,10}=7.428 ; P=0.021$ for linear trend; $F_{1,10}=13,711 ; P=0.004$ for cubic trend). However, pairwise comparison of foraging times obtained with this device revealed no statistically significant differences between day 1 (127 $\pm 45 \mathrm{~min} /$ day) and day 7 (119 $\pm 53 \mathrm{~min} /$ day; paired $t$-test: $P>0.05)$. Thus, no evidence was found that parrots foraged less after seven days of experience with the enrichment items.

\section{Discussion}

The main goal of this study was to test the efficacy of eleven foraging enrichments to increase foraging times in Grey parrots. It was hypothesized that all enrichments would result in significant increases of foraging times compared to baseline, with puzzle feeders resulting in the highest increases in foraging times. In addition to testing the efficacy, we also evaluated the differential effects of the foraging enrichments on time allocation of foraging-related activities (i.e., locomotory, enrichmentand food-related behaviours), as well as the learning curves and possible habituation effects that may occur over time.

Foraging times increased significantly for nine out of eleven foraging enrichments compared to baseline values. However, differences were present in the extent to which the foraging enrichments increased foraging times. In addition, distinct differences were observed in the time allocation and learning curves for the different enrichments. These findings may help parrot owners and professionals dealing with parrots in their daily work (e.g., veterinarians, behaviourists, animal care takers) to gain further insight into the benefits and limitations of using specific types of enrichment to stimulate foraging behaviour in captive (Grey) parrots.

Captive Grey parrots spent on average $47 \pm 18$ min per day on foraging activities when offered a conventional pelleted diet in a regular food bowl. These baseline values are in accordance with those found in Amazon parrots (Oviatt and Millam, 1997; Rozek et al., 2010).

According to our expectations and similar to results in other species (e.g. Johnson et al., 2004; Skibiel et al., 2007; Gottlieb et al., 2011), provision of foraging enrichments led to an increase in the time spent on foraging. Increases were found to be significant for all but two enrichments, i.e. SD1 and ET3. Although these foraging enrichments scored the lowest foraging times $(68 \pm 27$, and $76 \pm 39 \mathrm{~min}$, respectively) of all tested enrichments, the lack of significance $(P=0.012$ and $P=0.017)$ may well be the result of the stringent conditions that were set for the statistical analysis. It has been argued that the Bonferroni correction that we applied to the results is too conservative and would stack the odds too strongly in favour of negative findings (e.g. Nakagawa, 2004). A post hoc power analysis of the available data, however, revealed that approximately 30 parrots would be needed for these two enrichments to demonstrate a statistical significant difference $(P<0.0045)$ in foraging time compared to baseline, which is a number far greater than the number of birds needed for the other enrichments.

Compared to the other enrichments, however, the SD1 and ET3 were also the least effective enrichments. The principle behind these two types of enrichment may account for their lower efficacy. For the SD1, the increase in foraging time primarily results from extra time spent on travelling from one food bowl to the other. Although this approach was shown effective in parrots housed in an aviary (e.g., Coulton and Young, 1997; van Hoek and King, 1997; Elson and Marples, 2001), the size of the cage in which our parrots were housed was so small that no more than 15 seconds were needed to travel from one side of the cage to the other. As a consequence, total time spent on locomotory behaviour was minimal $(5.4 \pm 4.6 \mathrm{~min} /$ day $)$. Thus, an enrichment approach based on increasing spatial distribution of food will likely only be effective when a parrot is housed in a large enclosure. For the ET3, the action needed to open the device is quite difficult, as indicated by the long learning curves and large number of parrots that were not able to master the technique. Once the parrot has learned how to open the device, however, only a short period of time (seconds to minutes) may be needed to unlock the device and gain access to all of the food. From this point onwards, the device is comparable to a regular food bowl and will thus hardly result in an increased feeding time compared to baseline. Foraging times with this enrichment were indeed ranked among the least effective enrichments. This finding, together with the significantly longer learning curve and large percentage of birds not being able to master the technique, renders the device (and similar devices) less suitable when the foraging enrichment is provided with the intention to increase foraging times.

For the two other puzzle feeders that were classified among the least-effective (i.e. ET4, ET5), the increases in 

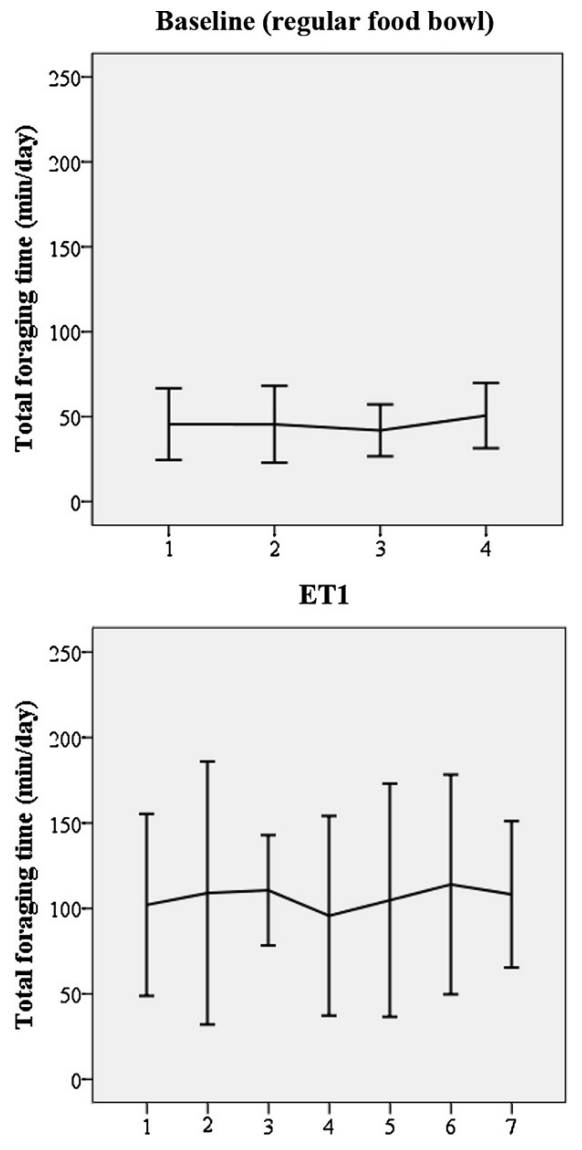

ET4

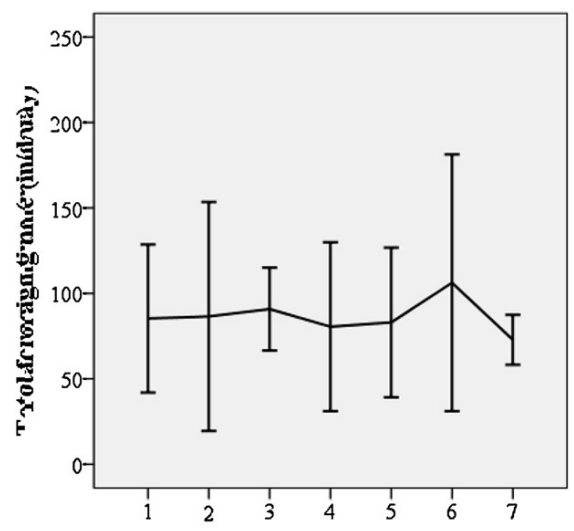

ET7

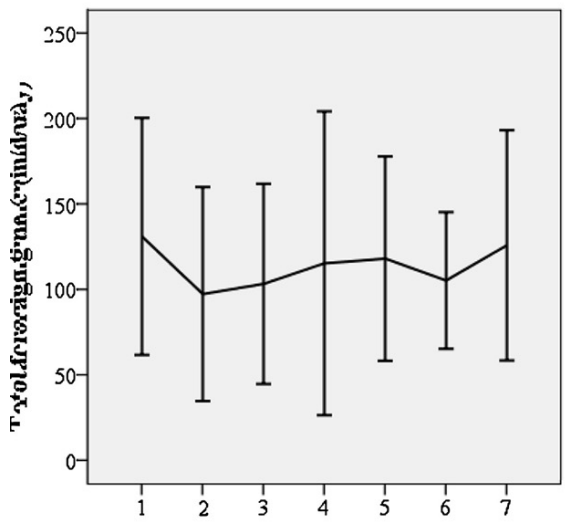

SD1 $(N=9)$

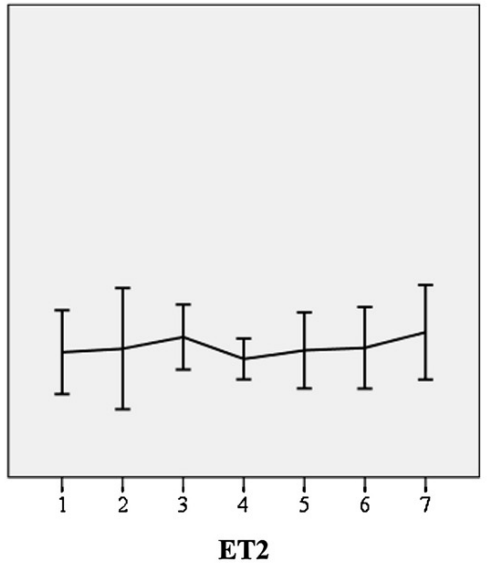

ST1
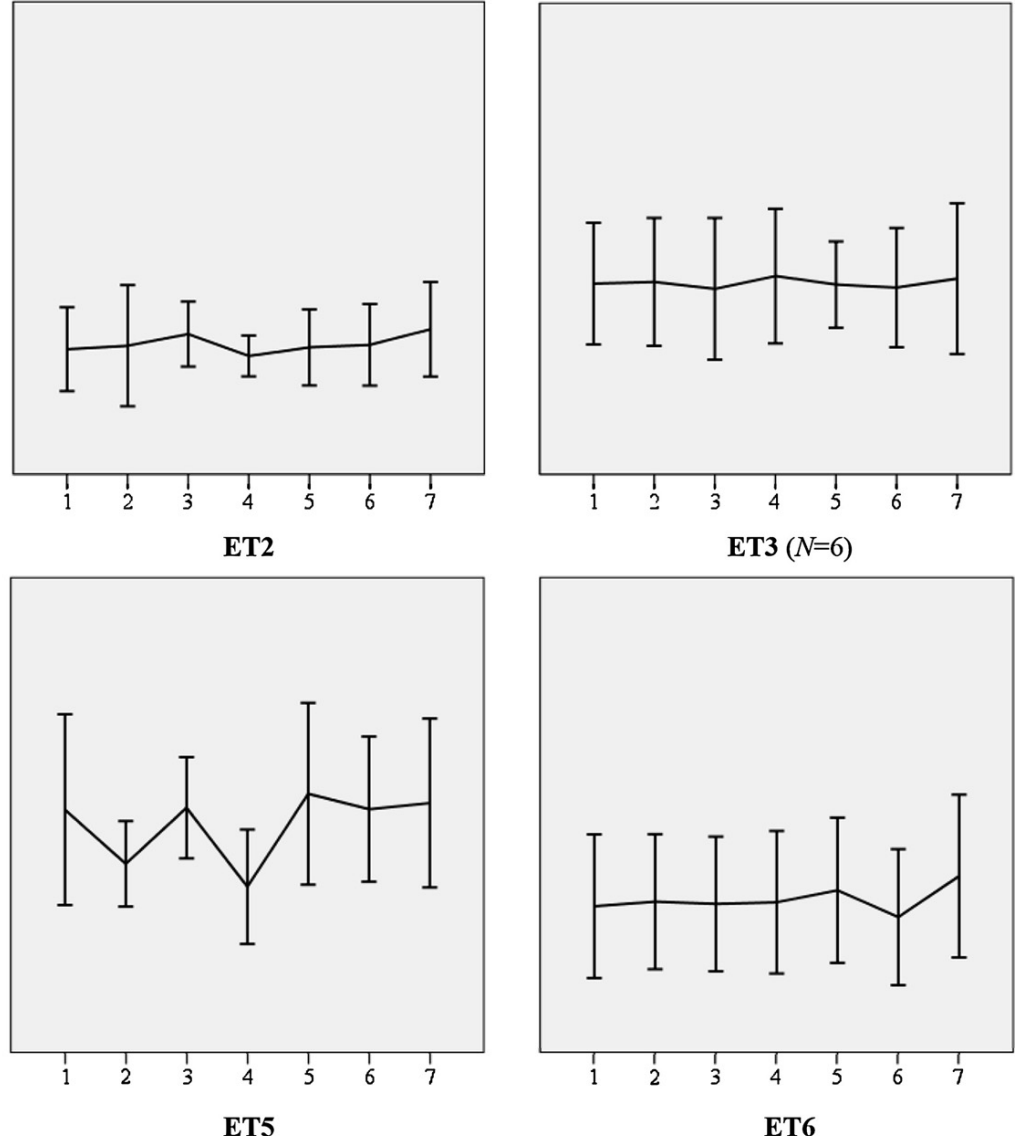

T6

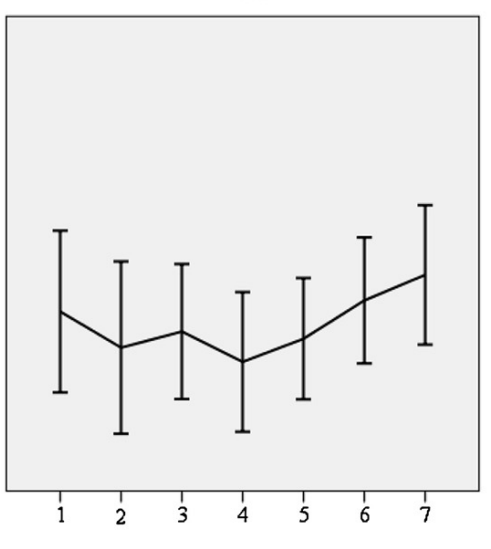

ET8
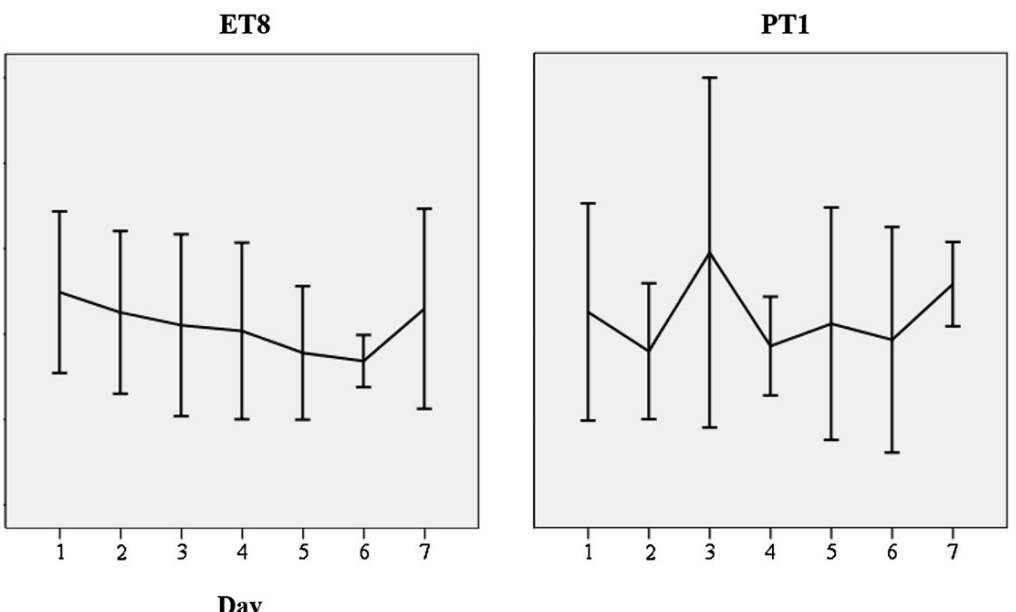

Fig. 4. Daily fluctuations in foraging times of grey parrots $(N=11)$ using the different foraging enrichments. 
foraging time compared to baseline were approximately two-fold. Although these increases were significant, they were still significantly less than the increases in foraging times obtained with the most-effective device (ET6). Again, this difference in efficacy may be due to the design and mechanism of the devices, which requires the bird to turn or spin the wheel in order to get food near the opening (or vice versa). As the opening in both devices is quite large it may be relatively easy to extract food from the devices, therefore only resulting in moderate increases of foraging times.

The most-effective enrichments (ET2, ET6 and ET7) were able to induce 2.5 -fold increases (up to $123 \pm 52 \mathrm{~min}$ ) in foraging time compared to baseline, and resulted in significantly longer foraging times compared to the leasteffective foraging enrichments (SD1, ET3, ET5). The high efficacy of the ET6 may be explained by the necessity of the parrots to tear and shred the cardboard prior to gaining access to the food. This type of activity, however, was also displayed independent of feeding. Similar to other intelligent species such as pigs and chimpanzees (Videan et al., 2005; Bracke, 2006), parrots were found to have a preference for chewable and destructible enrichments (van Hoek and King, 1997; Reed and Price, 2000; Kim et al., 2009; Webb et al., 2010). Destructible materials may serve as foraging substitutes, providing the parrot with an opportunity to display chewing and tearing behaviours that are part of normal foraging behaviour (Reed and Price, 2000; Kim et al., 2009). These characteristics, combined with the potency to induce 2.5-fold increases in foraging times render this type of puzzle feeder as one of the preferred types of enrichment to use.

Other foraging enrichments that appeared equally effective to the ET6 and resulted in average foraging times $>100 \mathrm{~min} /$ day were the ET2, ET7, ET8 and PT1. For two of these enrichments (ET7 and ET8), food needed to be obtained through smaller sized holes. As these holes were only slightly bigger than the pellets, the birds needed more time to extract pellets from these enrichments, hence resulting in longer feeding and therefore also longer foraging times. For the ET8, which resulted in similar increases of total foraging times compared to baseline ( $\sim 60 \mathrm{~min}$ ) as found previously (Lumeij and Hommers, 2008), the increase in time spent on food-related behaviours almost reached statistical significance $(P=0.008)$. The latter suggested that indeed more time was spent on extracting the pellets from the device. In contrast, only $9 \pm 16 \%$ was spent on direct interaction with the enrichment as no specific manipulations were necessary to obtain pellets from the openings.

For other devices (e.g., ET1, ET2, ET3, ET6, ET7) extra manipulations of the device (e.g., pulling a lever, unscrewing cups, shredding cardboard, lifting a lid, turning keys) were needed prior to gaining access to the food. Thus, increases in foraging time for these devices could be attributed for a great part to an increased amount of time spent on enrichment-related activities. Similarly, the approach aiming to increase search time (ST1) by mixing food with inedible items, also increased the time spent on both food- and enrichment-related activity. Apart from this interaction, aimed to obtain food, the parrots also regularly played with the marbles, holding them in their feet and manipulating them with the beak. Similarly to the cardboard material of the ET6, the marbles could be regarded as foraging substitutes.

The foraging enrichment that aimed to increase process time (PT1) resulted in similar foraging times (>100 min/day) as the most-effective puzzle feeders. PT1 consists of a nutritionally balanced mix of peanuts, hulled canary seed, cracked corn and pellets shaped into the form of a berry. Some may argue that the composition of this mixture, which includes seeds, may have influenced the results for this type of enrichment. It has previously been demonstrated, however, that parrots spend equal amounts of time foraging on seed mixtures and pelleted diets (Wolff et al., 2002). The increases in foraging times seen with the PT1 therefore seem to stem predominantly from offering the food as over-sized particles. Similarly, larger-sized pellets were found to significantly increase foraging times compared to regular-sized pellets (Rozek et al., 2010). Parrots had to break down these pellets in smaller pieces prior to being able to eat and swallow them. Although a similar principle applied to the PT1, the increase in time achieved with this enrichment was less than found by Rozek et al. (2010). As both food items were similar of size $(\sim 2.5 \mathrm{~cm}$ diameter), other diet-related factors (e.g., hardness, structure, nutritional composition and/or energy content of the food) may have contributed to this difference. To identify if and which factors play a role, further studies are needed.

The amount of food consumed for the enrichments ET8 $(53 \pm 5 \mathrm{~g})$ and PT1 (62 $\pm 9 \mathrm{~g})$ was significantly higher compared to the other enrichments. The most likely explanation for the measured increased food intake is that due to nibbling of pieces [PT1] or crumbling of pellets during extraction [ET8] more food was wasted, while the actual food intake was not increased. As the weight of the parrots remained stable throughout the study, the differences in food consumption were not considered clinically relevant.

In addition to the differences observed in total foraging times and time allocation to the different foraging-related activities, differences were also noted with regard to the time that was needed for the parrots to master a specific foraging technique. Although the foraging enrichments were gradually introduced and the time needed to master the foraging technique was recorded only when the parrot was acclimatized to the item in the cage, neophobia may still have played a role as fearful parrots may be less inclined to approach and manipulate the novel enrichment (e.g. Fox and Millam, 2007). The most likely cause for differences between learning curves for the different enrichments therefore appears to be the differences in difficulty level of the enrichments. Various enrichmentrelated factors may have influenced this difficulty level, e.g. the complexity of the behaviour which needed to be learned, resemblance between the action needed and natural behaviour, and/or ability of the bird to see where the food was located (i.e. transparent versus opaque materials). For the two devices that had the longest learning curves (i.e. ET2, ET3) a more difficult and/or unnatural action (unscrewing a cup, turning a key) was required, in addition to one of the enrichments (ET2) also being opaque. In contrast, parrots needed little to no time to learn how to 
use SD1 and PT1, as parrots only needed to learn where the food was located and no further effort was required to obtain the food. Although these findings suggest that the aforementioned factors may influence the difficulty level of a specific type of enrichment, further studies are required to systematically investigate whether and which of these factors are of major influence on the learning process.

No habituation effects were identified for any of the enrichments during the study. Other studies demonstrated that such habituation effects do occur in other species (e.g. Kuczaj et al., 2002; Tamura, 2011). However, the time frame needed to induce these habituation effects varied greatly, from less than a week (Kuczaj et al., 2002) to up to several weeks (Tamura, 2011). Thus, although no habituation occurred in the short-term, the time frame used in this study was too short for any conclusions to be drawn regarding long-term habituation effects to the enrichment. To keep the environment stimulating and prevent habituation effects, it has been recommended to regularly change the enrichment in the enclosure (Kuczaj et al., 2002; Shyne, 2006). Our findings suggest that enrichments do not need to be changed more frequent than once a week to remain their stimulating effect without resulting in habituation.

In this study we evaluated the effects of various enrichments on foraging times and activity. As the different enrichments all intend to a specific part of foraging activity, each of them may also exert a different efficacy with regard to reduction or prevention of abnormal behaviour. The effect of the different enrichments on welfare or abnormal behaviours, however, was not evaluated.

Several authors have suggested that feather damaging behaviour and stereotypies result from a lack of opportunity to forage (e.g. Keiper, 1969; Mason and Mendl, 1997; Huber-Eicher and Wechsler, 1998; Meehan et al., 2003; Lumeij and Hommers, 2008). Moreover, the type of stereotypy displayed may indicate which type of behaviour cannot be expressed sufficiently in the confined situation (Mason and Mendl, 1997), and thus also indicate which type of enrichment may be most successful in combating the problem. Locomotor stereotypies may benefit most from provision of enrichment aimed at increasing spatial or temporal distribution of food, or at increasing the search time. Oral stereotypies and feather damaging behaviour, on the other hand, may benefit more from enrichments that increase the time spent on procurement, manipulation and consumption of food. Although some studies have demonstrated the effect of foraging devices and destructible items on the reduction of feather damaging behaviour and oral stereotypies in parrots (Meehan et al., 2003; Lumeij and Hommers, 2008), further studies are needed to evaluate whether different foraging enrichments indeed exert a different effect on reduction of stereotypies.

Thus far, maximum foraging times that have been obtained in captive parrots provided with foraging enrichment did not exceed 3 hours per day (Elson and Marples, 2001; Lumeij and Hommers, 2008; Rozek et al., 2010). When comparing these times to those of wild conspecifics, i.e., 4-8 hours per day (Snyder et al., 1987), a time gap of at least 1-4hours is evident. Part of the foraging time in the wild is spent on flying to and from food patches. A greater proportion of this time, however, is spent on manipulation and consumption of food (e.g. Masello et al., 2006). Although foraging times obtained with the currently available foraging enrichment are significantly increased compared to baseline, they are still likely to be too short to naturalize foraging times of captive parrots. This may also explain why the provision of foraging enrichment reduces rather than eliminates feather damaging behaviour (Meehan et al., 2003; Lumeij and Hommers, 2008).

To further increase foraging times, currently available foraging enrichments may be refined, e.g. by decreasing the number and/or size of openings through which food can be extracted, or by adapting the devices to the specific needs and preferences of the bird. In previous studies, preferences for specific colours, sizes, hardness and structure have been evaluated in parrots (Kim et al., 2009; Webb et al., 2010; Rozek and Millam, 2011). These preferences may, however, differ between genders, ages and/or species. Further research will be needed to identify the preferred properties of a foraging device. In addition, attention should be paid to the prevent hunger and frustration which may occur when the bird is aware of the presence of food but is unable to obtain it. The rewarding properties of the foraging activity itself, as well as the frequency, consistency and contiguity with which the desired food is received during the process, should therefore also be taken into account when increasing foraging time to an optimum. Alternative to the refinement of existing devices, new, more effective types of foraging enrichment may be developed, in which the aforementioned properties and precautions are also taken into account.

\section{Conclusion}

This study demonstrates that foraging enrichments can effectively increase foraging times. Approaches focussing on increasing extraction time (by using puzzle feeders) and increasing food processing time (i.e., use of larger-sized food particles) appeared to be most effective to increase foraging time. As the types of enrichment used for these approaches specifically stimulate activities that involve beak manipulations, these may subsequently also be the ones that are preferred in the prevention and/or treatment of abnormal repetitive behaviours such as oral stereotypies and feather damaging behaviour. Further research will be necessary to identify whether this is indeed the case.

Although 2 to 2.5-fold increases in foraging times compared to baseline were achieved, none of the enrichments were able to naturalize foraging times. This finding emphasizes the necessity to develop new, more effective types of foraging enrichment. When developing such an enrichment, the parrots' preferences and time- and activity-enhancing properties of the device need to be taken into account in order to maximize the birds' opportunities to fulfil their behavioural need to forage.

\section{Acknowledgements}

The authors would like to thank Dr. H. de Vries for his help with the statistical analysis of the results. In addition, we would like to thank Lafeber company (Cornell, 
USA), Lorre \& Co (Wije, The Netherlands), Parrots Avenue (Sliedrecht, The Netherlands), and Parrotshop Nederland (Wilbertoord, The Netherlands) for kindly providing some of the enrichments that were used in this study.

This study was made possible with the help of a grant received from the Dutch Ministry of Economic affairs, Agriculture and Innovation (Grant No. 1400001780).

\section{References}

Aerni, V., El-Lethey, H., Wechsler, B., 2000. Effect of foraging material and food form on feather pecking in laying hens. Br. Poultry Sci. 41, $16-21$.

Baker, K.C., 1997. Straw and forage material ameliorate abnormal behaviors in adult chimpanzees. Zoo Biol. 16, 225-236.

Bashaw, M.J., Bloomsmith, M.A., Marr, M.J., Maple, T.L., 2003. To hunt or not to hunt? A feeding enrichment experiment with captive large felids. Zoo Biol. 22, 189-198.

Bauck, L., 1998. Psittacine diets and behavioral enrichment. Sem. Avian Exotic Pet Med. 7, 135-140.

Bloomsmith, M.A., Alford, P.L., Maple, T.L., 1988. Successful feeding enrichment for captive chimpanzees. Am. J. Primatol. 16, 155-164.

Birchall, A., 1990. Who's a clever parrot, then? New Scientist, February 24, pp. 38-43.

Bracke, M.B.M., 2006. Expert opinion regarding environmental enrichment materials for pigs. Anim. Welf. 15, 67-70.

Brinch-Riber, A., Mench, J.A., 2008. Effects of feed- and water-based enrichment on activity and cannibalism in Muscovy ducklings. Appl. Anim. Behav. Sci. 114, 429-440.

Brouns, F., Edwards, S.A., English, P.R., 1994. Effect of dietary fibre and feeding system on activity and oral behaviour of group housed sows. Appl. Anim. Behav. Sci. 39, 215-223.

Cannon, C., 1992. Proventricular and ventricular obstruction with bedding materials. J. Assoc. Avian Vet. 6, 40

Collier, G.H., Rovée-Collier, C.K., 1981. A comparative analysis of optimal foraging behavior: Laboratory Simulations. In: Kamil, A.C., Sargent, T.D. (Eds.), Foraging Behavior. Ecological, Ethological and Psychological Approaches. Garland STPM Press, New York, NY, USA, pp. 39-76.

Coulton, L.E., Waran, N.K., Young, R.J., 1997. Effects of foraging enrichment on the behaviour of parrots. Anim. Welf. 6, 357-363.

Crocket, C.M., 1998. Psychological well-being of captive nonhuman primates: lessons from laboratory studies. In: Shepherdson, D.J., Mellen, J.D., Hutchins, M. (Eds.), Second Nature: Environmental Enrichment for Captive Animals. Smithsonian Institution Press, Washington DC, USA, pp. 83-96.

Davis, C., 1998. Appreciating avian intelligence: the importance of a proper domestic environment. J. Am. Vet. Med. Assoc. 212, 1220-1222.

de Leeuw, J., 2004. Stimulation of behavioural and nutritional satiety in pigs. Ph.D. Thesis. Wageningen University.

Dixon, L.M., Duncan, I.J.H., Mason, G.J., 2010. The effects of four types of enrichment on feather-pecking behaviour in laying hens housed in barren environments. Anim. Welf. 19, 429-435.

Elson, H.L.G., Marples, N.M., 2001. How captive parrots react to foraging enrichments. In: Proc. 3rd Ann. Symp. Zoo Res, Chester, UK, pp. 1-8.

Fox, R.A., Millam, J.R., 2007. Novelty and individual differences influence neophobia in orange-winged Amazon parrots (Amazona amazonica). Appl. Anim Behav. Sci. 104, 107-115.

Francisco, M.R., Lunardi, V.O., Galetti, M., 2002. Massive seed predation of Pseudobombax grandiflorum (Bombacaceae) by parakeets Brotogeris versicolurus (Psittacidae) in a forest fragment in Brazil. Biotropica 34, 613-615.

Gilardi, J.D., Munn, C.A., 1998. Patterns of activity, flocking and habitat use in parrots of the Peruvian Amazon. Condor 100, 641-653.

Gottlieb, D.H., Ghirardo, S., Minier, D.E., Sharpe, N., Tatum, L., McCowan, B., 2011. Efficacy of 3 types of foraging enrichment for Rhesus macaques (Macaca mulatta). J. Am. Assoc. Lab. Anim. Sci. 50, 888-894.

Graham, D.L., 1998. Pet birds: historical and modern perspectives on the keeper and the kept. J. Am. Vet. Med. Assoc. 212, 1216-1219.

Hernandez-Divers, S.M., 2006. Diet presentation: enriching the environment of exotics and wildlife. In: Proc. N. Am. Vet. Conf, Orlando, FL, USA, pp. 1791-1793.

Honess, P.E., Marin, C.M., 2006. Enrichment and aggression in primates. Neurosci. Biobehav. Rev. 30, 413-436.

Huber-Eicher, B., Wechsler, B., 1998. The effect of quality and availability of foraging materials on feather pecking in laying hen chicks. Anim. Behav. 55, 861-873.
Hughes, B.O., Duncan, I.J.H., 1988. The notion of ethological 'need', models of motivation and animal welfare. Anim. Behav. 36, 1696-1707.

Inglis, I.R., Forkman, B., Lazarus, J., 1997. Free food or earned food? A review and fuzzy model of contrafreeloading. Anim. Behav. 53, 1171-1191.

Jensen, P., Toates, F.M., 1993. Who needs 'behavioural needs'? Motivational aspects of the needs of animals. Appl. Anim. Behav. Sci. 37, $161-181$.

Johnson, S.R., Patterson-Kane, E.G., Niel, L., 2004. Foraging enrichment for laboratory rats. Anim. Welf. 13, 305-312.

Joseph, L., 2010. Contrafreeloading and its benefits to avian behavior. In: Proc. Assoc. Avian Vet, San Diego, CA, USA, pp. 399-401.

Keiper, R.R., 1969. Causal factors of stereotypies in caged birds. Anim. Behav. 17, 114-119

Kim, L.C., Garner, J.P., Millam, J.R., 2009. Preferences of Orange-winged Amazon parrots (Amazona amazonica) for cage enrichment devices. Appl. Anim. Behav. Sci. 120, 216-223.

Kuczaj, S., Lacinak, T., Trone, M., Solangi, M., Ramos, J., 2002. Keeping environmental enrichment enriching. Int. J. Comp. Psychol. 15, $127-137$.

Lindqvist, C.E.S., Schütz, K.E., Jensen, P., 2002. Red jungle fowl have more contrafreeloading than white leghorn layers: effect of food deprivation and consequences for information gain. Behaviour 139, 1195-1209.

Lumeij, J.T., 1994. Gastroenterology. In: Ritchie, B.W., Harrison, G.J., Harrison, L.R. (Eds.), Avian Medicine: Principles and Application. Wingers Publishing Inc, Lake Worth, FL, pp. 482-521.

Lumeij, J.T., Hommers, C.J., 2008. Foraging 'enrichment' as treatment for pterotillomania. Appl. Anim. Behav. Sci. 111, 85-94.

Magrath, R.D., Lill, A., 1985. Age related differences in behavior and ecology of crimson rosellas during the non-breeding season. Austr. Wildl. Res. 12, 299-306.

Masello, J.F., Pagnossin, M.L., Sommer, C., Qullfeldt, P., 2006. Population size, provisioning frequency, flock size and foraging range at the largest known colony of Psittaciformes: The Burrowing Parrots of the north-eastern Patagonian coastal cliffs. Emu 106, 69-79.

Mason, G., Mendl, M., 1997. Do the stereotypies of pigs, chickens and mink reflect adaptive species differences in the control of foraging? Appl. Anim. Behav. Sci. 53, 45-58.

May, D.L., 2001. Grey parrots of the Congo basin forest. PsittaScene 13, $8-10$.

Meehan, C.L., Millam, J.R., Mench, J.A., 2003. Foraging opportunity and increased physical complexity both prevent and reduce psychogenic feather picking by young Amazon parrots. Appl. Anim. Behav. Sci. 80, 71-85.

Meehan, C.L., Garner, J.P., Mench, J.A., 2004. Environmental enrichment and development of cage stereotypy in Orange-winged Amazon parrots (Amazona amazonica). Dev. Psychobiol. 44, 209-218.

Miller, K.A., Mench, J.A., 2005. The differential effects of four types of environmental enrichment on the activity budgets, fearfulness, and social proximity preference of Japanese quail. Appl. Anim. Behav. Sci. 95, 169-187.

Morrisey, J.K., 1996. Gastrointestinal diseases of psittacine birds. Semin. Avian Exotic Pet. Med. 2, 66-74.

Nakagawa, S., 2004. A farewell to Bonferroni: the problems of low statistical power and publication bias. Behav. Ecol. 15, 1044-1045.

Newberry, R.C., 1995. Environmental enrichment: increasing the biological relevance of captive environments. Appl. Anim. Behav. Sci. 44, 229-243.

Ogura, T., 2011. Contrafreeloading and the value of control over visual stimuli in Japanese macaques (Macaca fuscata). Anim. Cogn. 14, 427-431.

Oviatt, L.A., Millam, J.R., 1997. Breeding behavior of captive Orangewinged Amazon parrots. Exotic Bird Rep. 9, 6-7.

Reed, H.K., Price, D.J., 2000. Environmental enrichment and the behaviour of hyacinth macaws at Paignton Zoo. In: Proc. 2nd Ann. Symp. Zoo Res, London, UK, pp. 89-94.

Renton, K., 2001. Lilac-crowned Parrot diet and food resource availability: resource tracking by a parrot seed predator. Condor 103, 62-69.

Rozek, J.C., Danner, L.M., Stucky, P.A., Millam, J.R., 2010. Over-sized pellets naturalize foraging time of captive Orange-winged Amazon parrots (Amazona amazonica). Appl. Anim. Behav. Sci. 125, 80-87.

Rozek, J.C., Millam, J.R., 2011. Preference and motivation for different diet forms and their effect on motivation for a foraging enrichment in captive Orange-winged Amazon parrots (Amazona amazonica). Appl. Anim. Behav. Sci. 129, 153-161.

Rushen, J., Lawrence, A.B., Terlouw, E.M.C., 1993. The motivational basis of stereotypies. In: Lawrence, A.B., Rushen, J. (Eds.), Stereotypic Animal Behavior: Fundamentals and Applications to Welfare. CAB International, Wallingford, United Kingdom, pp. 41-64. 
Shepherdson, D.J., Carlstead, K., Mellen, J.D., Seidensticker, J., 1993. The influence of food presentation on the behavior of small cats in confined environments. Zoo Biol. 12, 203-216.

Shyne, A., 2006. Meta-analytic review of the effects of enrichment on stereotypic behavior in zoo mammals. Zoo Biol. 25, 317-337.

Skibiel, A.L., Trevino, H.S., Naugher, K., 2007. Comparison of several types of enrichments for captive felids. Zoo Biol. 26, 371-381.

Snyder, N.F.R., Wiley, J.W., Kepler, C.B., 1987. The Parrots of Luquillo: Natural History and Conservation of the Puerto Rican Parrot. Western Found. Vert. Zool, Los Angeles, CA, 384 p.

Spoolder, H.A.M., Burbidge, J.A., Edwards, S.A., Simmins, P.H., Lawrence, A.B., 1995. Provision of straw as a foraging substrate reduces the development of excessive chain and bar manipulation in food restricted sows. Appl. Anim. Behav. Sci. 43, 249-262.

Swaisgood, R.R., Shepherdson, D.J., 2005. Scientific approaches to enrichment and stereotypies in zoo animals: what's been done and where should we go next? Zoo Biol. 24, 499-518.

Symes, C.T., Perrin, M.R., 2003. Daily flight activity and flocking behaviour patterns of the Greyheaded Parrot (Poicephalus fuscicollis suahelicus) Reichenow 1898 in Northern Province, South Africa. Trop. Zool. 16, 47-62.

Tamura, N., 2011. Population differences and learning effects in walnut feeding technique by the Japanese squirrel. J. Ethol. 29, 351-363.

van Hoek, C.S., King, C.E., 1997. Causation and influence of environmental enrichment on feather picking of the crimson-bellied conure (Pyrrhura perlata perlata). Zoo Biol. 16, 161-172.

van Krimpen, M.M., Kwakkel, R.P., van der Peet-Schwering, C.M.C., den Hartog, L.A., Verstegen, M.W.A., 2009. Effects of nutrient dilution and nonstarch polysaccharide concentration in rearing and laying diets on eating behavior and feather damage of rearing and laying hens. Poult. Sci. 88, 759-773.

van Zeeland, Y.R.A., Schoemaker, N.J., Lumeij, J.T., 2009. Contrafreeloading in grey parrots. In: Proc. Assoc. Avian Vet, Milwaukee, WI, USA, p. 9.

Vargas-Ashby, H.W., Pankhurst, S.J., 2007. Effects of feeding enrichment on the behaviour and welfare of captive Waldrapps (Northern bald ibis) (Geronticus eremita). Anim. Welf. 16, 369-374.

Videan, E.N., Fritz, J., Schwandt, M.L., Smith, H.F., Howell, S, 2005. Controllability in environmental enrichment for captive chimpanzees (Pan troglodytes). J. Appl. Anim. Welfare Sci. 8, 117-130.

Webb, N.V., Famula, T.R., Millam, J.R., 2010. The effect of rope color, size and fray on environmental enrichment device interaction in male and female Orange-winged Amazon parrots (Amazona amazonica). Appl. Anim. Behav. Sci. 124, 149-156.

Westcott, D.A., Cockburn, A., 1988. Flock size and vigilance in Parrots. Austr. J. Zool. 36, 335-349.

Wirminghaus, J.O., Downs, C.T., Perrin, M.R., Symes, C.T., 2001. Abundance and activity patterns of the cape parrot (Poicephalus robustus) in two afromontane forests in South Africa. Afr. Zool. 36, 71-77.

Wolf, P., Graubohm, S., Kamphues, J., 2002. Experimental data on feeding extruded diets in parrots. In: Proc. Joint Nutr. Soc, Antwerp, Belgium, p. 137.

Young, R.J., 2003. Environmental enrichment for captive animals. UFAW Animal Welfare Series. Blackwell Publishers, Ames, Iowa, USA, 228 p.

Young, R.J., Carruthers, J., Lawrence, A.B., 1994. The effect of a foraging device (the "Edinburgh Foodball") on the behaviour of pigs. Appl. Anim. Behav. Sci. 39, 237-247. 